\title{
The minimum of a branching random walk outside the boundary case
}

\author{
JULIEN BARRAL ${ }^{1, *}$, YUEYUN HU ${ }^{1, * *}$ and THOMAS MADAULE ${ }^{2}$ \\ ${ }^{1}$ Département de Mathématiques, Université Paris XIII, Sorbonne Paris Cité, 99 avenue J-B Clément, \\ F-93430 Villetaneuse,France.E-mail: *barral@math.univ-paris13.fr; ${ }^{* *}$ yueyun@math.univ-paris13.fr \\ ${ }^{2}$ Institut de Mathématiques de Toulouse, Université Paul Sabatier, 118 route de Narbonne, F-31062 \\ Toulouse Cedex 9, France. E-mail: thomas.madaule@math.univ-toulouse.fr
}

This paper is a complement to the studies on the minimum of a real-valued branching random walk. In the boundary case [Electron. J. Probab. 10 (2005) 609-631], Aïdékon in a seminal paper [Ann. Probab. 41 (2013) 1362-1426] obtained the convergence in law of the minimum after a suitable renormalization. We study here the situation when the log-generating function of the branching random walk explodes at some positive point and it cannot be reduced to the boundary case. In the associated thermodynamics framework, this corresponds to a first-order phase transition, while the boundary case corresponds to a second-order phase transition.

Keywords: branching random walk; minimal position; phase transition

\section{Introduction}

\subsection{Model and main result}

Consider a branching random walk on the real line $\mathbb{R}$. Initially, a particle sits at the origin. Its children form the first generation; their displacements from the origin correspond to a point process $\mathscr{L}$ on the line. These children have children of their own (who form the second generation), and behave, relative to their respective positions, like independent copies of $\mathscr{L}$, and so on. Denote by $\mathbb{P}$ the probability distribution on the space $\Omega$ of marked trees associated with this branching random walk, and $\mathbb{E}$ the expectation with respect to $\mathbb{P}$.

The genealogy of all particles forms a Galton-Watson tree $\mathbb{T}$ whose root is denoted by $\varnothing$. Denote by $\{u:|u|=n\}$ the set of particles at generation $n \in \mathbb{N}$ and by $V(u) \in \mathbb{R}$ the position of $u$. Notice that $\sum_{|u|=1} \delta_{\{V(u)\}}=\mathscr{L}$. Denote by $v:=\sum_{|u|=1} 1$ the offspring number. Let $\phi$ be the log-generating function of $\mathscr{L}$ :

$$
\phi(\beta):=\log \mathbb{E}\left[\sum_{|u|=1} \mathrm{e}^{-\beta V(u)}\right]=\log \mathbb{E}\left[\int_{\mathbb{R}} \mathrm{e}^{-\beta x} \mathscr{L}(\mathrm{d} x)\right] \in(-\infty, \infty], \quad \beta \in \mathbb{R} .
$$

We assume that $\mathscr{L}$ is not almost surely supported on a deterministic lattice. Therefore, $\phi$ is strictly convex over its domain $\operatorname{dom}(\phi):=\{\beta, \phi(\beta)<\infty\}$ if $\operatorname{dom}(\phi)$ is non-trivial.

We assume that $\mathbb{T}$ is supercritical and define $M_{n}:=\min _{|u|=n} V(u)$ the minimum of the branching random walk in the $n$th generation (with the convention $\min \varnothing \equiv \infty$ ). 
Hammersley [27], Kingman [30] and Biggins [9] have established the law of large numbers for $M_{n}$ under a fairly general setting: if $\left\{\beta>0: \mathbb{E}\left[\sum_{|u|=1} \mathrm{e}^{-\beta V(u)}\right]<+\infty\right\} \neq \varnothing$ then conditioned upon the survival of the system, $\lim _{n \rightarrow \infty} \frac{M_{n}}{n}=v$, where $v=-\inf \{\phi(\beta) / \beta: \beta>0\}$. Hammersley [27] raised the problem of finding the asymptotic behavior of $M_{n}-v n$. Due to the convexity property of $\phi$, if $\inf \{\phi(\beta) / \beta: \beta>0\}$ is attained at $0<\beta_{v}<\infty$ and $\phi<\infty$ on a left neighborhood of $\beta_{v}$ (this is the case for instance when $v$ has a finite expectation), we have either $\phi\left(\beta_{v}\right) / \beta_{v}=\phi^{\prime}\left(\beta_{v}\right)$ or $\phi\left(\beta_{v}\right) / \beta_{v}>\phi^{\prime}\left(\beta_{v}\right)$ and $\phi(\beta)=\infty$ for $\beta>\beta_{v}$, so that after making the change of variable $(V(u), u \in \mathbb{T}) \rightarrow\left(\beta_{v} V(u)+\phi\left(\beta_{v}\right)|u|, u \in \mathbb{T}\right)$, we can assume that either $\phi(1)=1$ and $\phi^{\prime}(1-)=0$, or $\phi(1)=0, \phi^{\prime}(1-)<0$, and $\phi(\beta)=\infty$ for all $\beta>1$.

Several recent attempts to solve Hammersley's problem led to significant contributions (see $[1,17,28]$ and the references therein), until the sharp answer was given by Aïdékon in [2] in the "boundary case" (terminology introduced in [14]) which corresponds to $\phi(1)=\phi^{\prime}(1-)=0$.

We study here the asymptotic behavior of $M_{n}$ outside the boundary case, that is, when

$$
\begin{aligned}
\phi(1) & =0, \\
\phi^{\prime}(1-) & <0 \text { and } \phi(1+)=\infty .
\end{aligned}
$$

In the associated thermodynamics framework, this corresponds to a first-order phase transition, while the boundary case corresponds to a second-order phase transition; see the forthcoming Section 1.2 for detailed discussions.

For our purpose, it will be convenient to state our assumptions in terms of the distribution of the i.i.d. increments $X_{1}, \ldots, X_{n}, \ldots$ of the random walk $\left(S_{n}\right)$ naturally associated with the branching random walk and assumed to be defined on a probability space whose probability measure is $\mathbf{P}$. Denote by $\mathbf{E}$ the expectation with respect to $\mathbf{P}$ and set $X=X_{1}$. The law of $X$, denoted as $\mathbf{P}_{X}$, is defined under (1.1) by

$$
\int_{\mathbb{R}} f(x) \mathbf{P}_{X}(\mathrm{~d} x):=\mathbb{E}\left[\sum_{|u|=1} f(V(u)) \mathrm{e}^{-V(u)}\right],
$$

for any bounded measurable function $f$. Observe that for all $\beta, \phi(\beta)=\log \int_{\mathbb{R}} \mathrm{e}^{-(\beta-1) x} \mathbf{P}_{X}(\mathrm{~d} x)$. Then a sufficient condition for $\phi(1+)=\infty$ is that near to $-\infty, \mathbf{P}_{X}(\mathrm{~d} x)$ has a density which decays polynomially or subexponentially. In this paper, we only treat the polynomial decay case.

Our assumptions about $\mathbf{P}_{X}$ and expressed in terms of $X$ are the following: There exist some constants $\gamma>3, \alpha>1$, a slowly varying function $\ell$ and some $x_{0}<0$ such that

$$
\begin{aligned}
\mathrm{m}: & =\mathbf{E}[X]>0, \quad \mathbf{E}\left[\left(X^{+}\right)^{\gamma}\right]<\infty, \\
\mathbf{P}(X \leq x) & =\int_{-\infty}^{x}|y|^{-\alpha-1} \ell(y) \mathrm{d} y \quad \forall x \leq x_{0},
\end{aligned}
$$

with $y^{+}:=\max (y, 0)$ for any $y \in[-\infty, \infty)$. We remark that the condition (1.4) implies (1.2): The first property $m>0$ is just a restatement of $\phi^{\prime}(1-)<0$ whenever this derivative is defined. The second and third properties imply in particular that $X$ is in the domain of attraction of a stable law of index $\min (\alpha, 2)$. One naturally gets a branching random walk leading 
to such an $X$ as follows: fix a random variable $X$ obeying (1.4) and assume in addition that $1<s=\mathbf{E}\left[\mathrm{e}^{X}\right]=\int \mathrm{e}^{x} \mathbf{P}_{X}(\mathrm{~d} x)<\infty$ (in particular the second condition holds with all $\gamma>0$ ). Let $\left(\xi_{j}\right)_{j \geq 1}$ be a sequence of random variables distributed according to $s^{-1} \mathrm{e}^{x} \mathbf{P}_{X}(\mathrm{~d} x)$, not necessarily independent. Let $v$ be a random integer independent of $\left(\xi_{j}\right)_{j \geq 1}$ such that $\mathbb{E}[v]=s$, and set $\mathscr{L}=\sum_{j=1}^{\nu} \delta_{\left\{\xi_{j}\right\}}$. When $s$ is an integer, $v$ can be taken constant and equal to $s$, so that the branching random walk is built on the $s$-adic tree.

For brevity, we extend the function $\ell$ to the whole $\mathbb{R}$, by letting $\ell(x)=\ell(-x)$ for $x \geq\left|x_{0}\right|$ and $\ell(x)=1$ for any $x \in\left(x_{0},\left|x_{0}\right|\right)\left[\left|x_{0}\right|\right.$ being large enough so that $\ell(x)>0$ for any $\left.x \leq x_{0}\right]$.

Under (1.1), it is known that on the set $\mathbf{S}$ of the survival of the system, $M_{n} \rightarrow \infty$ a.s. (see, e.g., Shi [43]).

It is natural to study the convergence in law of $M_{n}$ after renormalization. Under the condition (1.1), we introduce the martingale

$$
W_{n}:=\sum_{|u|=n} \mathrm{e}^{-V(u)}, \quad n \geq 1,
$$

which is called the additive martingale in the literature on the studies of branching random walks. Let $W_{\infty}$ be the almost sure limit of the non-negative martingale $W_{n}$. The following integrability hypothesis, which combined with $\mathbf{E}[X]>0$, is necessary and sufficient for $W_{\infty}$ to not vanish almost surely $[9,24,29]$ :

$$
\mathbb{E}\left[\left(\sum_{|u|=1} \mathrm{e}^{-V(u)}\right)\left(\log \sum_{|u|=1} \mathrm{e}^{-V(u)}\right)^{+}\right]<\infty,
$$

moreover $W_{\infty}>0$ on $\mathbf{S}$.

To establish the convergence in law for $M_{n}$, it is necessary to assume some additional conditions. To avoid the technical difficulties, we state below our result in the i.i.d. case, namely $\mathscr{L}$ can be written as $\mathscr{L}=\sum_{i=1}^{v} \delta_{\left\{\xi_{i}\right\}}$ with $\left(\xi_{i}\right)$ i.i.d. and independent of $v$.

Theorem 1.1 (i.i.d. case). Assume (1.1), (1.4) and (1.5). Suppose that $\mathscr{L}=\sum_{i=1}^{v} \delta_{\left\{\xi_{i}\right\}}$ with $\left(\xi_{i}\right)$ i.i.d. and independent of $v$. Then for any $x \in \mathbb{R}$,

$$
\lim _{n \rightarrow \infty} \mathbb{P}\left(M_{n} \geq \alpha_{n}+x\right)=\mathbb{E}\left(\exp \left(-c_{*} \mathrm{e}^{x} W_{\infty}\right)\right)
$$

where $\alpha_{n}:=(\alpha+1) \log n-\log \ell(n)$ and $c_{*}$ is a finite positive constant given as follows:

$$
c_{*}:=\mathrm{m}^{-(\alpha+1)} \sum_{j=0}^{\infty} \mathbb{E}\left[\mathrm{e}^{-M_{j}}\right] .
$$

We mention that the finiteness of $c_{*}$ is part of the proof of Theorem 1.6. 
Remark 1.2. In the i.i.d. case, namely when $\mathscr{L}=\sum_{i=1}^{v} \delta_{\left\{\xi_{i}\right\}}$ with $\left(\xi_{i}\right)$ i.i.d. and independent of $v,(1.1),(1.4)$ and (1.5) are equivalent to the following set of assumptions:

$$
\left\{\begin{array} { l } 
{ \mathbb { E } [ v ] > 1 , } \\
{ \mathbb { E } [ v \operatorname { l o g } \nu ] < \infty , }
\end{array} \text { and } \left\{\begin{array}{lc}
\mathbb{E}\left[\mathrm{e}^{-\xi}\right]=\frac{1}{\mathbb{E}[\nu]}, \quad \mathbb{E}\left[\xi \mathrm{e}^{-\xi}\right]>0, & \mathbb{E}\left[\left(\xi^{+}\right)^{\gamma} \mathrm{e}^{-\xi}\right]<\infty, \\
\mathbb{P}(\xi \in \mathrm{d} x)=\frac{1}{\mathbb{E}[\nu]}|x|^{-\alpha-1} \ell(x) \mathrm{e}^{-x} \mathrm{~d} x, & \text { for } x \leq x_{0},
\end{array}\right.\right.
$$

with $\alpha>1, \gamma>3$, a slowly varying function $\ell$ and $\xi:=\xi_{1}$.

Example 1.3. To fix ideas, let us give a concrete family of examples. Fix $\alpha>1, p \in(0,1)$, $x_{0}<0, x_{0}^{\prime}>0$ and let $\xi$ be a random variable with law equal to $\mu_{p}=p \delta_{x_{0}^{\prime}}+(1-p) \mu$, where $\mu((-\infty, x])=C_{\alpha, x_{0}} 1_{\left\{x \leq x_{0}\right\}} \mathrm{e}^{-|x|}|x|^{-\alpha-1}+1_{\left\{x>x_{0}\right\}}$, with $C_{\alpha, x_{0}}:=\mathrm{e}^{\left|x_{0}\right|}\left|x_{0}\right|^{\alpha+1}$.

By construction, we have $\mathbb{E}\left[\mathrm{e}^{-\beta \xi}\right]=\infty$ if $\beta>1$. Moreover,

$$
\begin{aligned}
& \mathbb{E}\left[\mathrm{e}^{-\xi}\right]=p \mathrm{e}^{-x_{0}^{\prime}}+(1-p) \mathrm{e}^{\left|x_{0}\right|}\left(1+\frac{\left|x_{0}\right|}{\alpha}\right) \text { and } \\
& \mathbb{E}\left[\xi \mathrm{e}^{-\xi}\right]=p x_{0}^{\prime} \mathrm{e}^{-x_{0}^{\prime}}-(1-p) \mathrm{e}^{\left|x_{0}\right|}\left(\frac{1+\alpha}{\alpha}\left|x_{0}\right|+\frac{1}{\alpha-1}\right) .
\end{aligned}
$$

Let

$$
p_{0}=\frac{\mathrm{e}^{\left|x_{0}\right|}\left(1+\left|x_{0}\right| / \alpha\right)-1}{\mathrm{e}^{\left|x_{0}\right|}\left(1+\left|x_{0}\right| / \alpha\right)-\mathrm{e}^{-x_{0}^{\prime}}} \in(0,1) .
$$

For all $p \in\left(p_{0}, 1\right)$, we have $\mathbb{E}\left[\mathrm{e}^{-\xi}\right]<1$. Moreover, if $x_{0}^{\prime}$ is chosen large enough, then the increasing function $p \in\left(p_{0}, 1\right) \mapsto \mathbb{E}\left[\xi \mathrm{e}^{-\xi}\right]$ vanishes at some point, say $\widetilde{p}_{0}$. Now, let $v$ be a random integer with expectation $\mathbb{E}\left[\mathrm{e}^{-\xi}\right]^{-1}$ and such that $\mathbb{E}[v \log v]<\infty$. For each $p \in\left(p_{0}, 1\right)$, let $\xi_{i}$, $i \geq 1$, be independent random variables distributed according to $\mu_{p}$, and also independent of $v$. They generate a point process $\sum_{i=1}^{v} \delta_{\left\{\xi_{i}\right\}}$ which is outside the boundary case when $p \in\left(\widetilde{p}_{0}, 1\right)$ (and all the properties gathered in Remark 1.2 hold), in the boundary case when $p=\widetilde{p}_{0}$, and reducible to the boundary case when $p \in\left(p_{0}, \widetilde{p}_{0}\right)$.

Remark 1.4. The general case will be treated in the Appendix. For instance, if $\mathscr{L}=\sum_{i=1}^{v} \delta_{\left\{\xi_{i}\right\}}$ with all $\xi_{i}=\xi$ a.s. for any $i \geq 1$, and $\xi$ independent of $v$, then (1.6) still holds (with some appropriate constant $c_{*}$ ).

Remark 1.5. In the boundary case, we have $\mathbf{E}[X]=0$, and the almost sure limit of the martingale $W_{n}$ vanishes (Biggins [10], Lyons [33]). Assuming that $\mathbf{E}\left[X^{2}\right]<\infty$ and some additional integrability conditions, Aïdékon [2] proved a highly non-trivial universal result (see also [16] for an alternative approach), namely the convergence in law for $M_{n}-\frac{3}{2} \log n$ as $n \rightarrow \infty$ toward a convoluted Gumbel distribution; specifically there exists a constant $c^{\prime}>0$ depending on the distribution of $\mathscr{L}$ such that

$$
\lim _{n \rightarrow \infty} \mathbb{P}\left(M_{n} \geq \frac{3}{2} \log n+x\right)=\mathbb{E}\left(\exp \left(-c^{\prime} \mathrm{e}^{x} D_{\infty}\right)\right) \quad \forall x \in \mathbb{R},
$$


where $D_{\infty}:=\lim _{n \rightarrow \infty} \sum_{|u|=n} V(u) \mathrm{e}^{-V(u)}$ is the non-trivial and non-negative limit of the socalled derivative martingale $[2,13,19]$. Theorem 1.1 exhibits another universality class with the Gumbel distribution convoluted by $W_{\infty}$ as limiting law.

Let us now say a few words on the normalizing sequence $\alpha_{n}$ and the additional conditions such as the i.i.d. case. For any $u \in \mathbb{T} \backslash\{\varnothing\}$, let $\overleftarrow{u}$ be the parent of $u$. Define

$$
\Delta V(u):=V(u)-V(\overleftarrow{u}), \quad \mathbb{B}(u):=\{v: v \neq u, \overleftarrow{v}=\overleftarrow{u}\}
$$

For any $n \geq 1$ and $|u|=n$, denote by $\left\{u_{0}:=\varnothing, u_{1}, \ldots, u_{n-1}, u_{n}=u\right\}$ the shortest path relating the root $\varnothing$ to $u$ such that $\left|u_{i}\right|=i$ for any $0 \leq i \leq n$.

It turns out that the minimal position $M_{n}$ will be reached only by those particles $|u|=n$, such that there is a unique $i \in[1, n]$ such that $\Delta V\left(u_{i}\right)<-n^{1+o(1)}$. Moreover, to make $V(u)=M_{n}$, necessarily $i$ is near to $n$ and this large drop $\Delta V\left(u_{i}\right)$ will be of order $-n$, which in view of the density function of $X$ in (1.4) happens with probability of order $\mathrm{e}^{-\alpha_{n}}$. This phenomenon is essential in the explanation of the normalizing constant $\alpha_{n}$. Moreover, the extra i.i.d. assumption in Theorem 1.1 guarantees that with overwhelming probability, no $v \in \mathbb{B}\left(u_{i}\right)$ can make a large drop in the sense that $\Delta V(v)<-n^{1+o(1)}$, which in turns implies that all particles $|w|=n$ such that $V(w)=M_{n}$ are necessarily descendants of $u_{i}$. This is the crucial point for the convergence of $M_{n}-\alpha_{n}$ in the i.i.d. case.

However, in the general case, some particles $v \in \mathbb{B}\left(u_{i}\right)$ could also make a large drop; furthermore, $v$ could also produce some descendants which reach $M_{n}$ in the $n$th generation. To get the convergence in law of $M_{n}-\alpha_{n}$, we have to control this possibility of simultaneous large drops in the same generation and the relative displacements between the particles in $\mathbb{B}\left(u_{i}\right)$ and $u_{i}$ itself. In particular, we need to assume the convergence in law of these relative displacements conditioned on $V\left(u_{i}\right) \rightarrow-\infty$, which is our main additional hypothesis in the general case. We refer to the Appendix for more details.

We end this subsection by a remark when the density of $\mathbf{P}_{X}(\mathrm{~d} x)$ in (1.4) decays subexponentially near to $-\infty$.

Remark 1.6. Assume that as $x \rightarrow-\infty, \mathbf{P}_{X}(\mathrm{~d} x) / \mathrm{d} x \sim c^{\prime}|x|^{a} \mathrm{e}^{-c|x|^{b}}$ for some constants $c>$ $0, c^{\prime}>0$ and $a \in \mathbb{R}, 0<b<1$. We conjecture that $M_{n}$ is of order $n^{b}$. Furthermore, if $0<b<\frac{1}{2}$, then we believe that the phenomenon of the unique large drop still holds which would lead to the tightness of $M_{n}-c(\mathrm{~m} n)^{b}+a \log n$.

\subsection{Connection with thermodynamics}

Due to the interplay between branching random walk theory and some random energy models in statistical physics, we find it useful to relate the above-mentioned fine results on $M_{n}$ to critical phenomena. In the setting of the previous section, that is, when $v=0$ and $\beta_{v}=1$, define the convex functions

$$
F_{n}(\beta)=\frac{1}{n} \log \sum_{|u|=n} \mathrm{e}^{-\beta V(u)}, \quad n \geq 1, \beta>0 .
$$



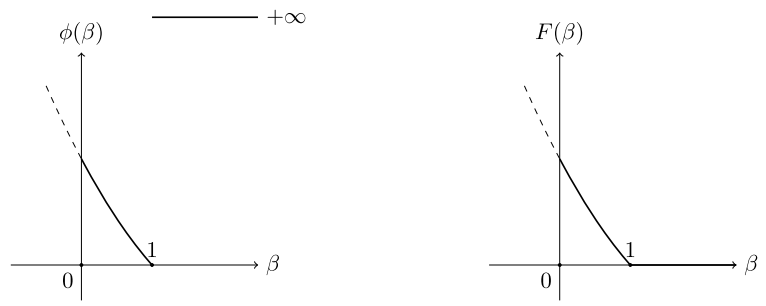

Figure 1. First-order phase transition.

In the random energy model introduced by Derrida and Spohn in [22] (in which $\mathbb{T}$ is a regular tree and the increments of the branching random walks are i.i.d. and Gaussian, so that only the boundary case occurs), these functions are the free energies of the directed polymers on the disordered tree $\mathbb{T}$. They converge almost surely pointwise on $\mathbb{R}_{+}$to the free energy in infinite volume

$$
F(\beta)=\mathbf{1}_{[0,1]}(\beta) \phi(\beta)+0 \cdot \mathbf{1}_{(1, \infty)}(\beta), \quad \beta>0
$$

(see $[4,11,20,39,41])$. Suppose that $\phi^{\prime}(1-)$ exists. The free energy $F$ is analytic over its domain except at 1 . In the boundary case $\phi^{\prime}(1-)=0, F$ is only once differentiable; in the thermodynamical setting this corresponds to a second-order phase transition at the inverse temperature $\beta=1$. When $\phi^{\prime}(1-)<0$ and $\phi(1+)=\infty, F$ is continuous and non-differentiable; we face a first-order phase transition at $\beta=1$ (see Figures 1 and 2 for illustrations where $\phi(0)$ is assumed finite).

Next, we recall some known facts associated with the boundary case, and we provide conjectures regarding analogous results associated with the case considered in this paper.

Aïdékon's result (1.8) is a key point in understanding the asymptotic behavior of the Gibbs measures $\mu_{\beta, n}$ which assigns to each bond $u$ of generation $n$ the mass $\mu_{\beta, n}(u)=\mathrm{e}^{-\beta V(u)-n F_{n}(\beta)}$. Based on [2], Madaule [35] showed that $n^{(3 / 2) \beta} \sum_{|u|=n} \mathrm{e}^{-\beta V(u)}$ converges in law; see also Webb [47] in the Gaussian case on a regular tree. In the case where $\mathbb{T}$ is regular, say $s$-adic, Barral, Rhodes and Vargas [8] showed, thanks to [35] and the theory of invariant distributions by random weighted means (also called fixed points of the smoothing transformation theory) [3,9,12,24,29, 32,38], that for each $\beta>1, \mu_{\beta, n}$ converges in law to a random discrete measure $\mu_{\beta}$ defined as follows: Let $\mu$ be the critical Mandelbrot measure on $\{0, \ldots, s-1\}^{\mathbb{N}_{+}}$associated with the
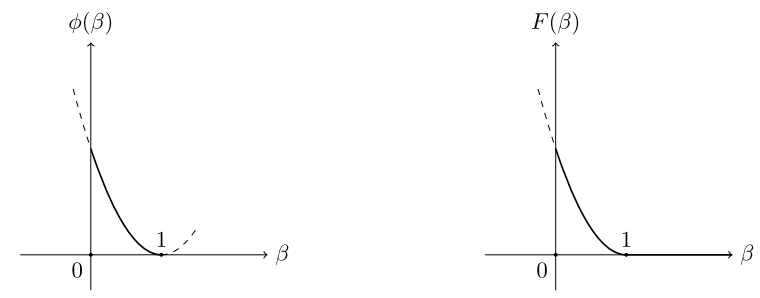

Figure 2. Second-order phase transition (boundary case). 
branching random walk, that is, the measure which assigns mass $\mathrm{e}^{-V(u)} D_{\infty}(u)$ to bond $u$, where $D_{\infty}(u)$ is the copy of $D_{\infty}$ built with the branching random walk rooted at $u$; let $N_{\mu}^{(\beta)}$ be a positive Borel random measure on $\{0, \ldots, s-1\}^{\mathbb{N}_{+}} \times \mathbb{R}_{+}^{*}$ whose law conditionally on $\mu$ is that of a Poisson point measure with intensity $\frac{\mu(\mathrm{d} x) \mathrm{d} z}{z^{1+1 / \beta}}$; then define the random measures $v_{\beta}(A)=$ $\int_{A} \int_{\mathbb{R}_{+}^{*}} z N_{\mu}^{(\beta)}(\mathrm{d} x, \mathrm{~d} z)$ and $\mu_{\beta}=v_{\beta} /\left\|v_{\beta}\right\|$. All these results provide a sharp description of the asymptotic behavior of the associated directed polymer at temperatures lower than the critical freezing temperature $\beta=1$. In particular, when the temperature goes to 0 they describe how the main part of the energy concentrates on a small number of atoms.

The convergence (1.8) and some of the previous results have been extended to the context of log-correlated Gaussian fields [15,23,36,37], which confers them an additional degree of universality.

Let us also mention that $M_{n}$ plays a role in the study of the modulus of continuity of the 0-dimensional measure $\mu$ [6].

Our result makes us conjecture that outside the boundary case, for $\beta>1, \mathrm{e}^{\beta \alpha_{n}} \sum_{|u|=n} \mathrm{e}^{-\beta V(u)}$ converges in law as $n \rightarrow \infty$; furthermore this would imply that the same convergence result as in the boundary case holds for the Gibbs measures $\mu_{\beta, n}$ on $\{0, \ldots, s-1\}^{\mathbb{N}_{+}}$if one replaces the critical Mandelbrot measure by the standard Mandelbrot measure, namely the non-degenerate measure which assigns mass $\mathrm{e}^{-V(u)} W_{\infty}(u)$ to bond $u$. Such results would complete the parallel between the freezing phenomena observed under a second- and a first-order phase transition.

Also, it is important to try and test the universality of such properties for instance by studying their validity in the context of more general log-Lévy multiplicative chaos $[5,7,25,42]$ than the Gaussian case, for which there is a natural formulation of the notion of being in or outside the boundary case.

Let us finish with a geometric description of the difference between second- and first-order phase transition at the critical inverse temperature $\beta=1$, and conditionally on non-extinction: under a second-order phase transition, there exists a minimal supporting subtree $\mathbb{T}(0)$ for the free energy in the sense that the bonds of generation $n$ in $\mathbb{T}$ which mainly contribute to the free energy $F_{n}(1)$ are those $u$ of $\mathbb{T}(0) \cap \mathbb{T}_{n}$; moreover, one observes the behavior, or singularity, $\frac{V(u)}{n} \approx 0=$ $-\phi^{\prime}(1)$ for the potential $V$ along $\partial \mathbb{T}(0)$, and $\# \mathbb{T}(0) \cap \mathbb{T}_{n} \approx \mathrm{e}^{o(n)}$. These properties are reminiscent of the fact that in the infinite volume limit $\partial \mathbb{T}(0)$ is of Hausdorff dimension 0 and such that $\lim _{n \rightarrow \infty} \frac{1}{n} \log \sum_{|u|=n,[u] \cap \partial \mathbb{T}(0) \neq \varnothing} \mathrm{e}^{-V(u)}=F(1)=0$, with $\lim _{n \rightarrow \infty} \frac{V\left(x_{\mid n}\right)}{n}=0$ for all $x \in \partial \mathbb{T}(0)$, where $x_{\mid n}$ is the prefix of $x$ of length $n$ and $\partial \mathbb{T}$ is endowed with the standard ultrametric distance. Consequently, the free energy concentrates on a single type of singularity (see $[4,40])$. Under a first order phase transition, for all $\alpha \in\left[0,-\phi^{\prime}(1)\right]$, there exists a subtree $\mathbb{T}(\alpha)$ of $\mathbb{T}$ such that $\# \mathbb{T}(\alpha) \cap \mathbb{T}_{n} \approx \mathrm{e}^{n \alpha}$, the bonds $u \in \mathbb{T}(\alpha) \cap \mathbb{T}_{n}$ satisfy $\frac{V(u)}{n} \approx \alpha$, and they substantially contribute to the free energy $F_{n}(1)$; in the infinite volume the fractal sets $\partial \mathbb{T}(\alpha), \alpha \in\left[0,-\phi^{\prime}(1)\right]$, are of respective Hausdorff dimension $\alpha$, and such that $\lim _{n \rightarrow \infty} \frac{1}{n} \log \sum_{|u|=n,[u] \cap \partial T(\alpha) \neq \varnothing} \mathrm{e}^{-V(u)}=F(1)=0$, and at each $x \in \partial \mathbb{T}(\alpha)$ one observes the singularity $\lim _{n \rightarrow \infty} \frac{V\left(x_{\mid n}\right)}{n}=\alpha$ (see [4] for more details). This can be interpreted as the coexistence of uncountably many equilibrium states in the system at $\beta=1$.

The next section is an outline of the proof of Theorem 1.1. 


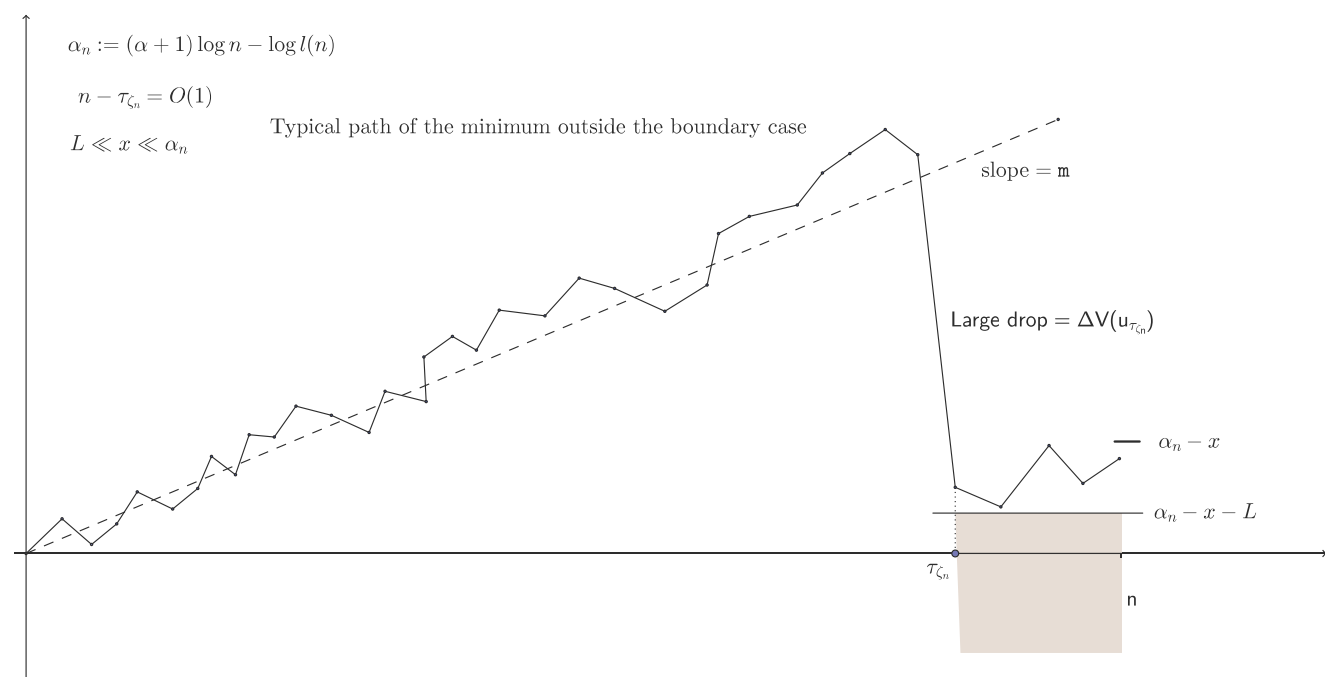

Figure 3. Typical path of an extremal particle.

\section{Outline of the proof of Theorem 1.1}

The main estimate leading to Theorem 1.1 is the following asymptotic tail for $M_{n}-\alpha_{n}$.

Proposition 2.1 (i.i.d. case). Assume (1.1), (1.4), (1.5). Suppose that $\mathscr{L}=\sum_{i=1}^{v} \delta_{\left\{\xi_{i}\right\}}$ with $\left(\xi_{i}\right)$ i.i.d. and independent of $\nu$. For any $\varepsilon>0$, there exist $A=A(\varepsilon)>0$ and an integer $n_{0}=n_{0}(\varepsilon)$ such that for all $n>n_{0}$ and $x \in\left[A, \frac{n}{\log n}\right]$,

$$
\left|\mathbb{P}\left(M_{n} \leq \alpha_{n}-x\right)-c_{*} \mathrm{e}^{-x}\right| \leq \varepsilon \mathrm{e}^{-x},
$$

where as before, $\alpha_{n}:=(\alpha+1) \log n-\log \ell(n)$ and $c_{*}>0$ is the constant defined in (1.7).

Remark 2.2. In the general case, under some additional assumptions (see (A.1), (A.2) and (A.3) in the Appendix), Proposition 2.1 still holds with the same normlization sequence $\alpha_{n}$ and some positive constant $c$ instead of $c_{*}$.

It turns out that the machinery developed by Aïdékon in [2] is general enough to be adapted in the case considered in this paper. As a matter of fact, the proof of Proposition 2.1 (of which Proposition 4.1 is one of the main ingredients) goes in the same spirit as that of Proposition 1.3 in Aïdékon [2], namely the localization of the trajectory of a particle $u$ such that $V(u)=M_{n}$. The main difference is that, while in the boundary case such a trajectory typically corresponds to an excursion of length $n$, in our situation the trajectory $\left(V\left(u_{j}\right), 0 \leq j \leq n\right)$ grows linearly until some generation $k$, near to $n$, where it makes a very large drop $\Delta V\left(u_{k}\right)$. Moreover, we can prove that $n-k=O(1), \Delta V\left(u_{k}\right)=-(\mathrm{m}+o(1)) n$ and in the i.i.d. case that no brothers of $u_{k}$ make a 
drop of size $-(\mathrm{m}+o(1)) n$ [Intuitively, $\left(V\left(u_{j}\right), 0 \leq j \leq n\right)$ has a unique large drop and this large drop can only happen near to $n$ thanks to Lemma 4.2].

Specifically, let us fix the threshold $\zeta_{n}:=\frac{n}{(\log n)^{3}}$. For any $u \in \mathbb{T}$, let $\tau_{\zeta_{n}}^{(u)}$ be the time of the first large drop in the path $\left\{V\left(u_{i}\right), 1 \leq i \leq|u|\right\}$ :

$$
\tau_{\zeta_{n}}^{(u)}:=\inf \left\{1 \leq i \leq|u|: \Delta V\left(u_{i}\right)<-\zeta_{n}\right\},
$$

with $\inf \varnothing:=\infty$. Under the assumptions (1.1) and (1.4), we analyze the particles leading to $M_{n}$ and obtain the following statement (see (5.1)): Let $L$ and $T$ be large constants. For all large $n$ and for a fixed $x>0$, we consider the event $\left\{\exists|u|=n: V(u) \leq \alpha_{n}-x\right\}$. By Lemma 4.3 ((4.6) and (4.8)), we may restrict our attention to those $|u|=n$ whose trajectory $\left(V\left(u_{j}\right), 0 \leq j \leq n\right)$ has a unique large drop, say at generation $k \equiv \tau_{\zeta_{n}}^{(u)}$. Moreover, $k \geq n-T$ (see Lemma 4.5) and $\min _{k \leq j \leq n} V\left(u_{j}\right) \geq \alpha_{n}-x-L$ with high probability (see Lemma 4.4). It follows that

$$
\begin{aligned}
\mathbb{P} & \left(M_{n} \leq \alpha_{n}-x\right) \\
= & \mathbb{E}\left[\frac{1}{\eta_{n}} \sum_{|u|=n} 1_{\left\{M_{n}=V(u) \leq \alpha_{n}-x\right\}}\right] \\
= & \mathbb{E}\left[\frac{1}{\eta_{n}} \sum_{|u|=n} 1_{\left\{M_{n}=V(u) \leq \alpha_{n}-x, \min _{\tau_{\zeta n}^{(u)} \leq j \leq n} V\left(u_{j}\right) \geq \alpha_{n}-x-L, \tau_{\zeta n}^{(u)} \in[n-T, n]\right\}}\right]+o(1) \mathrm{e}^{-x},
\end{aligned}
$$

where $\eta_{n}:=\sum_{|u|=n} 1_{\left\{V(u)=M_{n}\right\}}$ and $o(1) \rightarrow 0$ uniformly in $n$ and $x$, as $L, T \rightarrow \infty$.

The next step is to analyze the number $\eta_{n}$ and the event $\left\{M_{n}=V(u)\right\}$ in (2.2). To this end, we need to introduce the probability measure $\mathbb{Q}$ considered by Lyons [33] for general branching random walks (see also [46] for regular trees) and originally defined by Kahane and Peyrière [29] for regular trees and in the case where $W_{\infty}$ is non-degenerate $(\mathbb{Q}$ is there defined as the skew product of the probability $\mathbb{P}$ and the Mandelbrot measure $\mu$ [29] to study the Hausdorff dimension of $\mu$ ).

The following proposition is well known: Denote by $\left(\mathcal{F}_{n}, n \geq 0\right)$ the natural filtration of the branching random walk.

Proposition 2.3. Under (1.1), on the space $\widehat{\Omega}$ of marked trees enlarged by an infinite distinguished ray $\left(w_{n}, n \geq 0\right)$, called the spine, we may construct a probability measure $\mathbb{Q}$ such that:

(i) for any $n \geq 1$ and $|u|=n$, we have

$$
\left.\mathbb{Q} \circ \pi^{-1}\right|_{\mathcal{F}_{n}}:=\left.W_{n} \bullet \mathbb{P}\right|_{\mathcal{F}_{n}}, \quad \mathbb{Q}\left\{w_{n}=u \mid \pi^{-1}\left(\mathcal{F}_{n}\right)\right\}=\frac{\mathrm{e}^{-V(u)}}{W_{n}},
$$

where $\pi$ denotes the projection of $\widehat{\Omega}$ on $\Omega$;

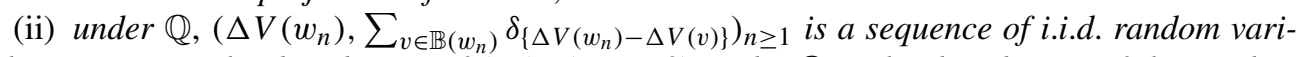
ables. Moreover, the distribution of $\left(V\left(w_{n}\right), n \geq 0\right)$ under $\mathbb{Q}$ is the distribution of the random walk $\left(S_{n}, n \geq 0\right)$ under $\mathbf{P}$ defined above;

(iii) under $\mathbb{Q}$, conditionally on $\mathcal{G}:=\sigma\left\{u, \Delta V(u), \overleftarrow{u}=w_{j}, j \geq 0\right\}$, the processes $\{V(u v)-$ $V(u), v \in \mathbb{T}\}$, for $u \in \bigcup_{j=1}^{\infty} \mathbb{B}\left(w_{j}\right)$, are i.i.d and are distributed as $\{V(v), v \in \mathbb{T}\}$ under $\mathbb{P}$. 
We refer the reader to $[13,18,33,34,43]$ for detailed discussions on the change of measure and the proof of Proposition 2.3.

We denote by $\mathbb{E}_{\mathbb{Q}}$ the expectation with respect to $\mathbb{Q}$. By the change of measure (Proposition 2.3), the expectation term in (2.2) is equal to

$$
\mathbb{E}_{\mathbb{Q}}\left[\frac{1}{\eta_{n}} \mathrm{e}^{V\left(w_{n}\right)} 1_{\left\{M_{n}=V\left(w_{n}\right) \leq \alpha_{n}-x, \min _{\tau_{\zeta n}^{\left(w_{n}\right)} \leq j \leq n} V\left(w_{j}\right) \geq \alpha_{n}-x-L, \tau_{\zeta n}^{\left(w_{n}\right)} \in[n-T, n]\right\}}\right] .
$$

Write $k:=\tau_{\zeta_{n}}^{\left(w_{n}\right)} \in[n-T, n]$. A crucial step in the localization of minimal particles, stated as Proposition 5.1, says that under (1.1), (1.4), (1.5) and the i.i.d. assumption, for any $|u|=n$ such that $V(u)=M_{n}$, necessarily $u_{k}=w_{k}$, that is, the trajectory of the particle $u$ and the spine coincide at least until the generation $k$. Consequently, both $\eta_{n}$ and $\left\{M_{n}=V\left(w_{n}\right)\right\}$ will only depend on the subtree rooted at $w_{k}$. By the Markov property of the branching random walk under the probability $\mathbb{Q}$, we get that

$$
\begin{aligned}
& \mathbb{P}\left(M_{n} \leq \alpha_{n}-x\right) \\
& \quad=\sum_{k=n-T}^{n} \mathrm{e}^{\alpha_{n}-x} \mathbb{E}_{\mathbb{Q}}\left[1_{\left\{\tau_{\zeta n}^{\left(w_{k}\right)}=k, V\left(w_{k}\right) \geq \alpha_{n}-x-L\right\}} F_{n-k}^{(L)}\left(V\left(w_{k}\right)-\left(\alpha_{n}-x-L\right)\right)\right]+o(1) \mathrm{e}^{-x},
\end{aligned}
$$

where $F_{j}^{(L)}$ is a measurable function defined in (5.9). Using the fact that under $\mathbb{Q},\left(V\left(w_{k}\right), k \geq 0\right)$ is distributed as the random walk $\left(S_{k}, k \geq 0\right)$, it follows that

$$
\begin{aligned}
& \mathbb{P}\left(M_{n} \leq \alpha_{n}-x\right) \\
& \quad=\mathrm{e}^{\alpha_{n}-x} \sum_{j=0}^{T} \mathbf{E}\left[1_{\left\{\tau_{\zeta_{n}}=n-j, S_{n-j} \geq \alpha_{n}-x-L\right\}} F_{j}^{(L)}\left(S_{n-j}-\left(\alpha_{n}-x-L\right)\right)\right]+o(1) \mathrm{e}^{-x} .
\end{aligned}
$$

Finally, we apply a renewal result (Lemma 3.6) and get Proposition 2.1 by letting $T, L \rightarrow \infty$. Theorem 1.1 follows from Proposition 2.1, exactly as the main result in Aïdékon [2] follows from an analogous, though different, proposition (pages 1405-1407). However, we give a proof for the reader's convenience.

Proof of Theorem 1.1 as a consequence of Proposition 2.1. For $B \geq 0$, define

$$
\mathcal{Z}[B]=\left\{u \in \mathbb{T}: V(u) \geq B, V\left(u_{k}\right)<B, \forall k<|u|\right\} .
$$

In the sense of [31], this is a very simple optional line and one has, by Theorem 9 in [31],

$$
\lim _{B \rightarrow \infty} \sum_{u \in \mathcal{Z}[B]} \mathrm{e}^{-V(u)}=W_{\infty}
$$

Fix $x \in \mathbb{R}$ and $\varepsilon \in\left(0, c_{*}\right)$. Let $A(\varepsilon) \geq 0$ and $n_{0}(\varepsilon)$ large be defined as in Proposition 2.1. Let $B>A(\varepsilon)+2|x|$ such that $\left(c_{*}+\varepsilon\right) \mathrm{e}^{-B / 2}<1$. Almost surely $\mathcal{Z}[B]$ is a finite subset of $\mathbb{T}$ (since 
$\lim _{n \rightarrow \infty} M_{n}=+\infty$ and the offspring number $v<\infty$ a.s.), then we can find $n_{1} \in \mathbb{N}_{+}$such that $n_{1} \geq n_{0}(\varepsilon)$ and

$$
\forall n \geq n_{1} \quad \mathbb{P}\left(\mathcal{Y}_{B, n}\right) \geq 1-\varepsilon,
$$

where

$$
\mathcal{Y}_{B, n}=\left\{V(u)-x \leq \frac{n}{\log n}, \forall u \in \mathcal{Z}[B]\right\} \cap\left\{\max \{|u|: u \in \mathcal{Z}[B]\} \leq n-n_{0}(\varepsilon)\right\} .
$$

Notice that by the choice of $B$, for any $u \in \mathcal{Z}[B], V(u)-x>A(\varepsilon)$. Now for $n \geq n_{1}$ we have

$$
\mathbb{P}\left(M_{n} \geq \alpha_{n}+x\right) \geq \mathbb{P}\left(M_{n} \geq \alpha_{n}+x, \mathcal{Y}_{B, n}\right)=\mathbb{E}\left(\mathbf{1}_{\mathcal{Y}_{B, n}} \prod_{u \in \mathcal{Z}[B]}\left(1-\Phi_{|u|, n}(V(u)-x)\right)\right),
$$

where we have used the conditional expectation along the stopping line and for $0 \leq k \leq n$, $\Phi_{k, n}(x):=\mathbb{P}\left(M_{n-k}<\alpha_{n}-x\right)$. By construction, we can apply Proposition 2.1 to each term of the product and get

$$
\mathbb{P}\left(M_{n} \geq \alpha_{n}+x\right) \geq \mathbb{E}\left(\mathbf{1}_{\mathcal{Y}_{B, n}} \prod_{u \in \mathcal{Z}[B]}\left(1-\left(c_{*}+\varepsilon\right) \mathrm{e}^{x-V(u)}\right)\right) .
$$

If necessary, we can enlarge $A(\varepsilon)$ to get $\forall u \in \mathcal{Z}[B], \mathrm{e}^{x-V(u)} \leq \mathrm{e}^{-A(\varepsilon)} \leq\left(c_{*}+\varepsilon\right)^{-1}$, so we have

$$
\mathbb{P}\left(M_{n} \geq \alpha_{n}+x\right) \geq \mathbb{E}\left(\prod_{u \in \mathcal{Z}[B]}\left(1-\left(c_{*}+\varepsilon\right) \mathrm{e}^{x-V(u)}\right)\right)-\mathbb{P}\left(\mathcal{Y}_{B, n}^{c}\right) .
$$

This yields

$$
\liminf _{n \rightarrow \infty} \mathbb{P}\left(M_{n} \geq \alpha_{n}+x\right) \geq \mathbb{E}\left(\prod_{u \in \mathcal{Z}[B]}\left(1-\left(c_{*}+\varepsilon\right) \mathrm{e}^{x-V(u)}\right)\right)-\varepsilon
$$

Moreover, since $\max \left\{\mathrm{e}^{-V(u)}: u \in \mathcal{Z}[B]\right\}$ tends a.s. to 0 as $B \rightarrow \infty$, we have $\lim _{B \rightarrow \infty} \sum_{u \in \mathcal{Z}[B]} \log \left(1-\left(c_{*}+\varepsilon\right) \mathrm{e}^{x-V(u)}\right)=-\left(c_{*}+\varepsilon\right) \mathrm{e}^{x} W_{\infty}$ hence by dominated convergence

$$
\liminf _{n \rightarrow \infty} \mathbb{P}\left(M_{n} \geq \alpha_{n}+x\right) \geq \mathbb{E}\left(\exp \left(-\left(c_{*}+\varepsilon\right) \mathrm{e}^{x} W_{\infty}\right)\right)-\varepsilon,
$$

and letting $\varepsilon$ tend to 0 yields the desired lower bound. To get the upper bound, write

$$
\mathbb{P}\left(M_{n} \geq \alpha_{n}+x\right) \leq \mathbb{P}\left(M_{n} \geq \alpha_{n}+x, \mathcal{Y}_{B, n}\right)+\mathbb{P}\left(\mathcal{Y}_{B, n}^{c}\right)
$$

Following the similar reasoning as above, we get

$$
\limsup _{n \rightarrow \infty} \mathbb{P}\left(M_{n} \geq \alpha_{n}+x\right) \leq \mathbb{E}\left(\prod_{u \in \mathcal{Z}[B]}\left(1-\left(c_{*}-\varepsilon\right) \mathrm{e}^{x-V(u)}\right)\right)+\varepsilon,
$$

and conclude as for the lower bound. 
The rest of the paper is organized as follows.

In Section 3, we collect some preliminary estimates on the one-dimensional random walk $\left(S_{n}\right)$. We prove Proposition 4.1 in Section 4, and Proposition 2.1 in Section 5 by admitting a localization Lemma 6.1. The proof of Lemma 6.1 is given in Section 6.

Throughout the text, we denote by $K, K^{\prime}$ and $K^{\prime \prime}$ possibly with several subscripts, some positive constants whose values may change from one paragraph to another one. We also wrote $f(n) \sim g(n)$ if $\lim _{n \rightarrow \infty} \frac{f(n)}{g(n)}=1$.

\section{Preliminaries on the one-dimensional random walk $\left(S_{n}\right)$}

Recall that we considered in the Introduction a sequence of i.i.d. real-valued random variables $\left(X_{i}\right)_{i \geq 1}$ distributed like $X$, and the random walk $\left(S_{n}\right)$ defined as $S_{n}:=S_{0}+X_{1}+\cdots+X_{n}$ for any $n \geq 1$ with $S_{0} \in \mathbb{R}$. Let $\bar{S}_{n}:=\max _{0 \leq k \leq n} S_{k}$ and $\underline{S}_{n}:=\min _{0 \leq k \leq n} S_{k}$. For $x \in \mathbb{R}$, denote the distribution of $\left(S_{n}\right)$ by $\mathbf{P}_{x}$ if $S_{0}=x$ and $\mathbf{P}=\mathbf{P}_{0}$. We state some known facts as lemmas.

Lemma 3.1 ([21], page 1950, Lemma 2.1). Let $\left(S_{n}\right)$ be a one-dimensional random walk satisfying $\mathbf{E}\left[\left|S_{1}\right|^{b}\right]<\infty$ for $b>1$. Let $\mathrm{m}:=\mathbf{E}\left[S_{1}\right]$. There exists a constant $K=K_{b}>0$ such that for all $n \geq 1, y \geq n^{\max (1 / b, 1 / 2)}$ and $x>0$,

$$
\begin{gathered}
\mathbf{P}\left(S_{n}-\mathrm{m} n \leq-x, \min _{1 \leq i \leq n} X_{i} \geq-y\right) \leq K \mathrm{e}^{-x / y}, \\
\mathbf{P}\left(\left|S_{n}-\mathrm{m} n\right| \geq x, \max _{1 \leq i \leq n}\left|X_{i}\right| \leq y\right) \leq K \mathrm{e}^{-x / y} .
\end{gathered}
$$

Lemma 3.2 (Gut [26], Theorem 6.2, page 93). Let $S$ be a one-dimensional random walk with positive mean $\mathrm{m}$ starting from 0 . Let

$$
R(x):=\sum_{n=0}^{\infty} \mathbf{P}\left(\bar{S}_{n} \leq x\right), \quad x \geq 0 .
$$

Then

$$
\lim _{x \rightarrow \infty} \frac{R(x)}{x}=\mathrm{m}
$$

Lemma 3.3 (Stone [44]). Assume (1.4). There exists a slowly varying function $\ell_{1}$ such that for all $x \in \mathbb{R}, \hbar>0$ and $n \geq 1$,

$$
\mathbf{P}\left(S_{n} \in[x, x+\hbar]\right) \leq \hbar n^{-\max (1 / \alpha, 1 / 2)} \ell_{1}(n) .
$$

We mention that up to a multiplicative constant, $\ell_{1}$ only depends on the truncated second moment of $S_{1}$; see Vatutin and Wachtel [45]. In particular, if $\alpha>2$, we may choose $\ell_{1} \equiv K$ for some large enough positive constant. 
Let us introduce the drop times in the random walk $\left(S_{n}\right)$ : for $\zeta>0$, define

$$
\begin{aligned}
\tau_{\zeta} & :=\inf \left\{j \geq 1: X_{j}<-\zeta\right\}, \\
\tau_{\zeta}^{(2)} & :=\inf \left\{j>\tau_{\zeta}: X_{j}<-\zeta\right\},
\end{aligned}
$$

the first and the second drop time of size $\zeta$. We shall consider $\zeta \in\left[\frac{\zeta_{n}}{4}, 4 \zeta_{n}\right]$ with

$$
\zeta_{n}:=\frac{n}{(\log n)^{3}}, \quad n \geq 2
$$

Lemma 3.4. Assume (1.4). There exists some constant $K>0$ such that for all $n \geq 2,-\infty<$ $y \leq \frac{\mathrm{m}}{2} n$

$$
\mathbf{P}\left(S_{n}-y \in[0,1]\right) \leq K n^{-\alpha} \ell(n)
$$

Proof. It is enough to consider large $n$. Observe that

$$
\begin{aligned}
\mathbf{P}\left(S_{n}-y \in[0,1]\right) \leq & \mathbf{P}\left(S_{n}-y \leq 1, \tau_{\zeta_{n}}>n\right)+\mathbf{P}\left(S_{n}-y \in[0,1], \tau_{\zeta_{n}}^{(2)} \leq n\right) \\
& +\sum_{i=1}^{n} \mathbf{P}\left(S_{n}-y \in[0,1], \tau_{\zeta_{n}}=i, \tau_{\zeta_{n}}^{(2)}>n\right) \\
= & : A_{(3.6)}+B_{(3.6)}+C_{(3.6)} .
\end{aligned}
$$

For any $y \leq \frac{m}{2} n$

$$
A_{(3.6)} \leq \mathbf{P}\left(S_{n}-\mathrm{m} n \leq 1-\frac{\mathrm{m}}{2} n, \tau_{\zeta_{n}}>n\right) \leq K \mathrm{e}^{-((\mathrm{m} / 2) n-1) / \zeta_{n}} \leq \mathrm{e}^{-(\mathrm{m} / 3)(\log n)^{3}},
$$

where we have applied Lemma 3.1 to get the second inequality in (3.7). For $B_{(3.6)}$, we deduce from Lemma 3.3 that

$$
\begin{aligned}
B_{(3.6)} & =\sum_{i=1}^{n-1} \sum_{j=i+1}^{n} \mathbf{P}\left(\tau_{\zeta_{n}}=i, \tau_{\zeta_{n}}^{(2)}=j, S_{n}-y \in[0,1]\right) \\
& \leq \sum_{i=1}^{n-1} \sum_{j=i+1}^{n} \mathbf{P}\left(\tau_{\zeta_{n}}=i, \tau_{\zeta_{n}}^{(2)}=j\right)(n-j+1)^{-\max (1 / \alpha, 1 / 2)} \ell_{1}(n-j+1) \\
& \leq n \times \zeta_{n}^{-2 \alpha} \ell\left(\zeta_{n}\right)^{2} n^{1-\max (1 / \alpha, 1 / 2)} \max _{1 \leq k \leq n} \ell_{1}(k)=o\left(n^{-\alpha}\right),
\end{aligned}
$$

since $2-\alpha-\max (1 / \alpha, 1 / 2)<0$. Finally, for all $n \geq 2$, let

$$
E_{i}^{(n)}:=\left\{\left|S_{n}-X_{i}-\mathrm{m}(n-1)\right| \leq \frac{n}{\log n}\right\}, \quad 1 \leq i \leq n .
$$


Observe that for any $\frac{\zeta_{n}}{4} \leq \zeta \leq 4 \zeta_{n}$ and $1 \leq i \leq n,{ }^{1}$

$$
\begin{aligned}
& \mathbf{P}\left(\tau_{\zeta}=i, \tau_{\zeta}^{(2)}>n,\left(E_{i}^{(n)}\right)^{c}\right) \\
& \quad=\mathbf{P}\left(\tau_{\zeta}=n,\left(E_{n}^{(n)}\right)^{c}\right) \\
& \quad=\mathbf{P}\left(X_{n}<-\zeta\right) \mathbf{P}\left(\min _{1 \leq j \leq n-1} X_{j} \geq-\zeta,\left|S_{n-1}-\mathrm{m}(n-1)\right|>\frac{n}{\log n}\right)
\end{aligned}
$$

Now by using a union bound and Markov's inequality, we get

$$
\begin{aligned}
\mathbf{P}\left(\tau_{\zeta}\right. & \left.=i, \tau_{\zeta}^{(2)}>n,\left(E_{i}^{(n)}\right)^{c}\right) \\
\leq & \zeta^{-\alpha} \ell(\zeta)\left(\mathbf{P}\left(\max _{1 \leq j \leq n-1} X_{j} \geq \zeta\right)\right. \\
& \left.+\mathbf{P}\left(\max _{1 \leq j \leq n-1}\left|X_{j}\right| \leq \zeta,\left|S_{n-1}-\mathrm{m}(n-1)\right|>\frac{n}{\log n}\right)\right) \\
& \leq \zeta^{-\alpha} \ell(\zeta)\left(n \zeta^{-\gamma} \mathbf{E}\left[\left(X^{+}\right)^{\gamma}\right]+K \mathrm{e}^{-n /(\zeta \log n)}\right) \quad(\text { by using (1.4) and (3.2)) } \\
\leq & n^{-(\alpha+\gamma-1)} \ell_{2}(n)
\end{aligned}
$$

with some slowly varying function $\ell_{2}$. Using exchangeability,

$$
\begin{aligned}
C_{(3.6)} & =n \mathbf{P}\left(X_{n}<-\zeta_{n}, S_{n}-y \in[0,1], \tau_{\zeta_{n}}=n\right) \\
& \leq n^{-(\alpha+\gamma-2)} \ell_{2}(n)+n \mathbf{E}\left[\left.1_{E_{n}^{(n)}} \mathbf{P}\left(X_{n}+s-y \in[0,1], X_{n}<-\zeta\right)\right|_{s=S_{n-1}}\right],
\end{aligned}
$$

by using the independence of $X_{n}$ and $S_{n-1}$. Notice that

$$
\sup _{x \leq-(\mathrm{m} / 3) n} \frac{\ell(x)}{|x|^{\alpha+1}} \leq(1+o(1)) \ell(n)\left(\frac{\mathrm{m}}{3} n\right)^{-\alpha-1} \leq K \mathrm{e}^{-\alpha_{n}},
$$

by using Karamata's representation for the slowly varying function $\ell$. On $E_{n}^{(n)}, y-S_{n-1} \leq$ $-\frac{m}{2} n+\mathrm{m}+\frac{n}{\log n} \leq-\frac{\mathrm{m}}{3} n-1$. It follows from (1.4) and (3.10) that on $E_{n}^{(n)}$, uniformly for $s=$ $S_{n-1}, \mathbf{P}\left(X_{n}+s-y \in[0,1]\right) \leq(1+o(1)) \ell(n)\left(\frac{m}{3} n\right)^{-\alpha-1}$, which implies that for all large $n$, $C_{(3.6)} \leq\left(\frac{\mathrm{m}}{3}\right)^{-\alpha-1}(1+o(1)) n^{-\alpha} \ell(n)$. Lemma 3.4 follows from (3.6).

Recall that $\alpha_{n}=(\alpha+1) \log n-\log \ell(n)$.

\footnotetext{
${ }^{1}$ We consider $\zeta$ instead of $\zeta_{n}$ for the use of (3.9) in the proof of Lemma 3.5; Moreover, by exchangeability, the probability $\mathbf{P}\left(\tau_{\zeta}=i, \tau_{\zeta}^{(2)}>n,\left(E_{i}^{(n)}\right)^{c}\right)$ does not depend on $i$.
} 
Lemma 3.5. Assume (1.4). There exist $K>0$ and a slowly varying function $\ell_{3} \geq 1$ such that for all large $n \geq n_{0}, \forall \zeta \in\left[\frac{\zeta_{n}}{4}, 4 \zeta_{n}\right], a \leq \frac{n}{\log n}$, we have that

$$
\begin{aligned}
& \mathbf{P}\left(S_{n}-y \in[a, a+1], \min _{\tau_{\zeta} \leq j \leq n} S_{j} \geq y, \tau_{\zeta}^{(2)} \leq n\right) \\
& \quad \leq R(a+1) n^{1-\max (1 / \alpha, 1 / 2)-2 \alpha} \ell_{3}(n) \quad \forall y \in \mathbb{R},
\end{aligned}
$$

whereas for all $-\infty<y<\frac{m}{2} n$,

$$
\mathbf{P}\left(S_{n}-y \in[a, a+1], \min _{\tau_{\zeta} \leq j \leq n} S_{j} \geq y, \tau_{\zeta} \leq n<\tau_{\zeta}^{(2)}\right) \leq K R(a+1) \mathrm{e}^{-\alpha_{n}}
$$

Moreover, for any $\varepsilon>0$, there exists some $\lambda=\lambda(\varepsilon)>0$ such that for all $-\infty<y<\frac{m}{2} n$,

$$
\mathbf{P}\left(S_{n}-y \in[a, a+1], \min _{\tau_{\zeta} \leq j \leq n} S_{j} \geq y,\left|S_{n}-S_{\tau_{\zeta}}\right|>\lambda, \tau_{\zeta} \leq n\right) \leq \varepsilon \mathrm{e}^{-\alpha_{n}} .
$$

Similar results hold if we replace the interval $[a, a+1]$ by $[a, a+\hbar]$ with an arbitrary positive constant $\hbar$.

Proof of Lemma 3.5. We shall prove that for any $1 \leq i<n$,

$$
\begin{aligned}
& \mathbf{P}\left(S_{n}-y \in[a, a+1], \min _{\tau_{\zeta} \leq j \leq n} S_{j} \geq y, \tau_{\zeta}=i, \tau_{\zeta}^{(2)} \leq n\right) \\
& \quad \leq R(a+1) i^{-\max (1 / \alpha, 1 / 2)} n^{-2 \alpha} \ell_{3}(n) \quad \forall y \in \mathbb{R},
\end{aligned}
$$

whereas for all $-\infty<y<\frac{m}{2} n$,

$$
\begin{aligned}
& \mathbf{P}\left(S_{n}-y \in[a, a+1], \min _{\tau_{\zeta} \leq j \leq n} S_{j} \geq y, \tau_{\zeta}=i, \tau_{\zeta}^{(2)}>n\right) \\
& \quad \leq K \mathbf{P}\left(\bar{S}_{n-i+1} \leq a+1\right) \mathrm{e}^{-\alpha_{n}}+n^{-(\alpha+\gamma-1)} \ell_{3}(n)
\end{aligned}
$$

Clearly, up to a multiplicative constant, (3.11) and (3.12) follow from (3.14) and (3.15) by taking the sum over $i \in 1,2, \ldots, n-1$.

Let us denote by $\mathbf{P}_{(3.14)}(i)$ the probability term in (3.14). By considering the time-reversed random walk $\left(S_{n}-S_{n-k}, 0 \leq k \leq n\right) \stackrel{(d)}{=}\left(S_{k}, 0 \leq k \leq n\right)$, we get that for any $1 \leq i \leq n-1$, $\left(S_{n}, \min _{\tau_{\zeta} \leq j \leq n} S_{j},\left\{\tau_{\zeta}=i<\tau_{\zeta}^{(2)} \leq n\right\}\right)$ has the same distribution as $\left(S_{n}, S_{n}-\bar{S}_{\sigma_{n}},\left\{\sigma_{n}=n-\right.\right.$ $\left.i+1>\tau_{\zeta}\right\}$ ), where $\sigma_{n}:=\max \left\{k \in[1, n], X_{k}<-\zeta\right\}$ (with the usual convention $\max \varnothing:=0$ ). It follows that

$$
\begin{aligned}
\mathbf{P}_{(3.14)}(i) & =\mathbf{P}\left(S_{n}-y \in[a, a+1], \bar{S}_{n-i+1} \leq S_{n}-y, \sigma_{n}=n-i+1>\tau_{\zeta}\right) \\
& \leq \mathbf{P}\left(S_{n}-y \in[a, a+1], \bar{S}_{n-i} \leq a+1, X_{n-i+1}<-\zeta, \tau_{\zeta}<n-i+1\right) \\
& =\mathbf{E}\left[1_{\left\{X_{n-i+1}<-\zeta, \tau_{\zeta}<n-i+1, \bar{S}_{n-i} \leq a+1\right\}} \mathbf{P}_{S_{n-i+1}}\left(S_{i-1}-y \in[a, a+1]\right)\right],
\end{aligned}
$$


by the Markov property at $n-i+1$. Set $g(i)=\sup _{z \in \mathbb{R}} \mathbf{P}_{z}\left(S_{i}-y \in[a, a+1]\right)$. We have

$$
\begin{aligned}
\mathbf{P}_{(3.14)}(i) & \leq g(i-1) \mathbf{P}\left(X_{n-i+1}<-\zeta, \tau_{\zeta}<n-i+1, \bar{S}_{n-i} \leq a+1\right) \\
& \leq g(i-1) \sum_{1 \leq j<n-i+1} \mathbf{P}\left(X_{n-i+1}<-\zeta, X_{j}<-\zeta, \bar{S}_{j-1} \leq a+1\right) \\
& =g(i-1) \mathbf{P}(X<-\zeta)^{2} \sum_{1 \leq j<n-i+1} \mathbf{P}\left(\bar{S}_{j-1} \leq a+1\right) \\
& \leq g(i-1) \zeta^{-2 \alpha} \ell(\zeta)^{2} R(a+1),
\end{aligned}
$$

for all large $n$. According to Stone's local limit theorem (Lemma 3.3), for $i \geq 2$ one has $g(i-1) \leq$ $i^{-\max (1 / \alpha, 1 / 2)} \ell_{1}(i)$, since $g(0) \leq 1$, this yields (3.14) as we shall choose

$$
\ell_{3}(n):=\max \left(\ell_{2}(n), 4^{2 \alpha}(\log n)^{6 \alpha} \max _{1 \leq i \leq n, \zeta_{n} / 4 \leq \zeta \leq 4 \zeta_{n}} \ell_{1}(i) \ell(\zeta)^{2}, 1\right),
$$

where $\ell_{2}(n)$ is the slowly varying function that appeared in (3.9).

To prove (3.15), we first establish an inequality implying that when $S_{n}=o(n)$, with high probability there is a unique large drop $X_{\tau_{\zeta}}$ before $n$ and the drop is of order of magnitude $-\mathrm{m} n$. Recall (3.8) for the definition of $E_{i}^{(n)}$. Define for any $i \in[1, n]$,

$$
\mathbf{P}_{(3.17)}(i):=\mathbf{P}\left(S_{n}-y \in[a, a+1], \min _{i \leq j \leq n} S_{j} \geq y, \tau_{\zeta}=i, \tau_{\zeta}^{(2)}>n, E_{i}^{(n)}\right) .
$$

In view of (3.9), (3.15) will follow if we can prove that

$$
\mathbf{P}_{(3.17)}(i) \leq K \mathbf{P}\left(\bar{S}_{n-i+1} \leq a+1\right) \mathrm{e}^{-\alpha_{n}} .
$$

By conditioning on $\sigma\left\{X_{j}, 1 \leq j \leq n, j \neq i\right\}$, we have that

$$
\begin{aligned}
\mathbf{P}_{(3.17)}(i) & \leq \mathbf{P}\left(S_{n}-\min _{i \leq j \leq n} S_{j} \leq a+1, S_{n}-y \in[a, a+1], E_{i}^{(n)}\right) \\
& =\mathbf{E}\left[\left.1_{\left\{S_{n}-\min _{i \leq j \leq n} S_{j} \leq a+1, E_{i}^{(n)}\right\}} \mathbf{P}\left(X_{i}+t-y \in[a, a+1]\right)\right|_{t=S_{n}-X_{i}}\right] .
\end{aligned}
$$

On $E_{i}^{(n)},|t-\mathrm{m}(n-1)| \leq \frac{n}{\log n}$, so $z \equiv a+1+y-t \leq-\frac{\mathrm{m}}{3} n$ for all large $n \geq n_{0}$ and uniformly for all $a \leq \frac{n}{\log n}$ and $y<\frac{m}{2} n$, hence it follows from (1.4) and (3.10) that

$$
\mathbf{P}_{(3.17)}(i) \leq K \mathrm{e}^{-\alpha_{n}} \mathbf{P}\left(S_{n}-\min _{i \leq j \leq n} S_{j} \leq a+1, E_{i}^{(n)}\right),
$$

which yields (3.18) by using the fact that $\mathbf{P}\left(S_{n}-\min _{i \leq j \leq n} S_{j} \leq a+1\right)=\mathbf{P}\left(\bar{S}_{n-i+1} \leq a+1\right)$. This completes the proof of (3.15).

Remark that in (3.19), if we replace the event $\left\{S_{n}-\min _{i \leq j \leq n} S_{j} \leq a+1\right\}$ by $\left\{\left|S_{n}-S_{i}\right|>\lambda\right\}$ with $\lambda>0$, then for any $i \in[1, n]$,

$$
\mathbf{P}\left(\left|S_{n}-S_{i}\right|>\lambda, S_{n}-y \in[a, a+1], E_{i}^{(n)}\right) \leq K \mathrm{e}^{-\alpha_{n}} \mathbf{P}\left(\left|S_{n}-S_{i}\right|>\lambda\right) .
$$


Denote by $\mathbf{P}_{(3.13)}$ the probability term in (3.13). Notice that by (3.11), the probability that the event in (3.13) holds together with $\left\{\tau_{\zeta}^{(2)} \leq n\right\}$ is bounded by $R(a+1) n^{1-\max (1 / \alpha, 1 / 2)-2 \alpha} \ell_{3}(n) \leq$ $\frac{\varepsilon}{4} \mathrm{e}^{-\alpha_{n}}$ for all large $n \geq n_{0}(\varepsilon)$. On the other hand, we deduce from (3.15) then Lemma 3.2 that for some large but fixed integer $k=k(\varepsilon, a)$,

$$
\begin{aligned}
& \mathbf{P}\left(S_{n}-y \in[a, a+1], \min _{\tau_{\zeta} \leq j \leq n} S_{j} \geq y, \tau_{\zeta}<n-k, \tau_{\zeta}^{(2)}>n\right) \\
& \quad \leq K \sum_{j=k}^{\infty} \mathbf{P}\left(\bar{S}_{j} \leq a+1\right) \mathrm{e}^{-\alpha_{n}}+n^{1-(\alpha+\gamma-1)} \ell_{3}(n) \\
& \quad \leq \frac{\varepsilon}{4} \mathrm{e}^{-\alpha_{n}}
\end{aligned}
$$

for all $n \geq n_{1}(\varepsilon)$ [recalling that $\gamma>3$ ]. Therefore,

$$
\begin{aligned}
\mathbf{P}_{(3.13)} \leq & \frac{\varepsilon}{2} \mathrm{e}^{-\alpha_{n}}+\mathbf{P}\left(S_{n}-y \in[a, a+1],\left|S_{n}-S_{\tau_{\zeta}}\right|>\lambda, n-k \leq \tau_{\zeta} \leq n<\tau_{\zeta}^{(2)}\right) \\
\leq & \frac{\varepsilon}{2} \mathrm{e}^{-\alpha_{n}}+(k+1) n^{-(\alpha+\gamma-1)} \ell_{2}(n) \\
& +\sum_{i=n-k}^{n} \mathbf{P}\left(S_{n}-y \in[a, a+1],\left|S_{n}-S_{\tau_{\zeta}}\right|>\lambda, \tau_{\zeta}=i, E_{i}^{(n)}\right),
\end{aligned}
$$

by applying (3.9) to $i=n, n-1, \ldots, n-k$. Since $\gamma>3,(k+1) n^{-(\alpha+\gamma-1)} \ell_{2}(n) \leq \frac{\varepsilon}{4} \mathrm{e}^{-\alpha_{n}}$, which in view of (3.20) imply that $\mathbf{P}_{(3.13)} \leq \frac{3 \varepsilon}{4} \mathrm{e}^{-\alpha_{n}}+K \sum_{j=0}^{k} \mathbf{P}\left(\left|S_{j}\right|>\lambda\right) \mathrm{e}^{-\alpha_{n}} \leq \varepsilon \mathrm{e}^{-\alpha_{n}}$, if we choose some $\lambda=\lambda(k, \varepsilon)$ large enough. This proves (3.13) and completes the proof of Lemma 3.5.

We present a renewal result associated to the random walk $\left(S_{n}\right)_{n \geq 0}$.

Lemma 3.6. Under (1.4). Let $G: \mathbb{R}_{+} \times \mathbb{R} \rightarrow \mathbb{R}$ be a measurable function such that for some $b>1$ and some positive constant $K>0$,

$$
\sup _{z \in \mathbb{R}}|G(x, z)| \leq K(1+x)^{-b} \quad \forall x \geq 0 .
$$

Assume furthermore that for any $x \in \mathbb{R}_{+}, \lim _{z \rightarrow-\infty} G(x, z)$ exists, and denote it by $G_{*}(x)$. Then

$$
\lim _{n \rightarrow \infty} \mathrm{e}^{\alpha_{n}} \mathbf{E}\left[1_{\left\{\tau_{\zeta}=n, S_{n} \geq y\right\}} G\left(S_{n}-y, X_{n}\right)\right]=\mathrm{m}^{-(\alpha+1)} \int_{0}^{\infty} G_{*}(x) \mathrm{d} x,
$$

uniformly on $|y| \leq \frac{n}{\log n}$ and $\frac{\zeta_{n}}{4} \leq \zeta \leq 4 \zeta_{n}$.

In the proof of Proposition 2.1 [i.i.d. case], we shall apply Lemma 3.6 to some function $F_{j}^{(L)}(x)$ which does not depend on $z$. We keep the possibility of dependence on $z$ in the lemma to deal with the general case of Proposition 2.1 (see Remark 2.2). 
Proof of Lemma 3.6. Without loss of generality, we may assume that $G$ takes non-negative values. Let $\varepsilon>0$ be small. Let $E_{n}^{(n)}:=\left\{\left|S_{n-1}-\mathrm{m}(n-1)\right| \leq \frac{n}{\log n}\right\}$ as in (3.8). By (3.9),

$$
\mathbf{P}\left(\tau_{\zeta}=n,\left(E_{n}^{(n)}\right)^{c}\right) \leq n^{-(\alpha+\gamma-1)} \ell_{2}(n) \leq \varepsilon \mathrm{e}^{-\alpha_{n}},
$$

for all large $n$. Let us denote by $\mathbf{E}_{(3.22)}$ the expectation term in (3.22). Then

$$
\mathrm{e}^{\alpha_{n}} \mathbf{E}_{(3.22)}=\mathrm{e}^{\alpha_{n}} \mathbf{E}\left[1_{\left\{\tau_{\zeta}=n, S_{n} \geq y\right\} \cap E_{n}^{(n)}} G\left(S_{n}-y, X_{n}\right)\right]+O(\varepsilon) .
$$

To deal with the above expectation term, we distinguish two situations according to the value of $S_{n}-y$ : Clearly,

$$
\begin{aligned}
& \mathrm{e}^{\alpha_{n}} \mathbf{E}\left[1_{\left\{\tau_{\zeta}=n, S_{n}-y \geq n / \log n\right\} \cap E_{n}^{(n)}} G\left(S_{n}-y, X_{n}\right)\right] \\
& \quad \leq \mathrm{e}^{\alpha_{n}} K\left(1+\frac{n}{\log n}\right)^{-b} \mathbf{P}\left(X_{n}<-\zeta\right) \\
& \quad=K\left(1+\frac{n}{\log n}\right)^{-b} \mathrm{e}^{\alpha_{n}} \int_{-\infty}^{-\zeta}|x|^{-\alpha-1} \ell(x) \mathrm{d} x \\
& \quad \leq \varepsilon,
\end{aligned}
$$

uniformly in $\zeta \in\left[\frac{\zeta_{n}}{4}, 4 \zeta_{n}\right]$ since $b>1$. If $0 \leq S_{n}-y<\frac{n}{\log n}$, then on the event $E_{n}^{(n)}, X_{n}=$ $S_{n}-S_{n-1}$ satisfies that $\left|X_{n}+\mathrm{m}(n-1)\right| \leq 3 \frac{n}{\log n}$ uniformly in $|y| \leq \frac{n}{\log n}$, a fortiori, $X_{n} \leq-\zeta$ so that $\tau_{\zeta} \leq n$. In view of (3.23) and (3.24), we get that

$$
\begin{aligned}
\mathrm{e}^{\alpha_{n}} \mathbf{E}_{(3.22)} & =\mathrm{e}^{\alpha_{n}} \mathbf{E}\left[1_{\left\{\tau_{\zeta}=n, 0 \leq S_{n}-y<n / \log n\right\} \cap E_{n}^{(n)}} G\left(S_{n}-y, X_{n}\right)\right]+O(\varepsilon) \\
& =\mathrm{e}^{\alpha_{n}} \mathbf{E}\left[1_{\left\{0 \leq S_{n}-y<n / \log n\right\} \cap E_{n}^{(n)}} G\left(S_{n}-y, X_{n}\right)\right]-R_{n}+O(\varepsilon),
\end{aligned}
$$

with

$$
R_{n}:=\mathrm{e}^{\alpha_{n}} \mathbf{E}\left[1_{\left\{\tau_{\zeta} \leq n-1,0 \leq S_{n}-y<n / \log n\right\} \cap E_{n}^{(n)}} G\left(S_{n}-y, X_{n}\right)\right] .
$$

By using the density of $X_{n}$, we see that for all large $n$,

$$
\begin{aligned}
\mathrm{e}^{\alpha_{n}} \mathbf{E} & {\left[1_{\left\{0 \leq S_{n}-y<n / \log n\right\} \cap E_{n}^{(n)}} G\left(S_{n}-y, X_{n}\right)\right] } \\
\quad=\mathbf{E} & {\left[1_{E_{n}^{(n)}} \int_{\left\{0 \leq S_{n-1}+z-y<n / \log n\right\}} G\left(S_{n-1}+z-y, z\right)\left(\mathrm{e}^{\alpha_{n}}|z|^{-\alpha-1} \ell(z)\right) \mathrm{d} z\right], }
\end{aligned}
$$

where we notice that $|z+\mathrm{m}(n-1)| \leq 3 \frac{n}{\log n}$, and hence $\mathrm{e}^{\alpha_{n}}|z|^{-\alpha-1} \ell(z)=\mathrm{m}^{-(\alpha+1)}+o(1)$ uniformly in $y$. Thus, we get that

$$
\begin{aligned}
\mathrm{e}^{\alpha_{n}} \mathbf{E}\left[1_{\left\{0 \leq S_{n}-y<n / \log n\right\} \cap E_{n}^{(n)}} G\left(S_{n}-y, X_{n}\right)\right] \\
\quad=\left(\mathrm{m}^{-(\alpha+1)}+o(1)\right) \mathbf{E}\left[1_{E_{n}^{(n)}} \int 1_{\left\{0 \leq S_{n-1}+z-y<n / \log n\right\}} G\left(S_{n-1}+z-y, z\right) \mathrm{d} z\right]
\end{aligned}
$$




$$
\begin{aligned}
& =\left(\mathrm{m}^{-(\alpha+1)}+o(1)\right) \mathbf{E}\left[1_{E_{n}^{(n)}} \int_{0}^{n / \log (n)} G\left(x, x+y-S_{n-1}\right) \mathrm{d} x\right] \\
& =:\left(\mathrm{m}^{-(\alpha+1)}+o(1)\right) \mathbf{E}_{(3.26) .}
\end{aligned}
$$

Notice that for any fixed $x \in \mathbb{R}_{+}$and $|y| \leq \frac{n}{\log n}, 1_{E_{n}^{(n)}} G\left(x, x+y-S_{n-1}\right)$ converges a.s. to $G_{*}(x)$ as $n \rightarrow \infty$. Indeed $-S_{n-1}$ tends linearly to $-\infty$ and $1_{E_{n}^{(n)}}$ converges to 1 a.s. by the Kolmogorov-Marcinkiewicz-Zygmund law of large numbers. It then follows from (3.21) and the dominated convergence theorem that $\mathbf{E}_{(3.26)} \rightarrow \int_{0}^{\infty} G_{*}(x) \mathrm{d} x$, uniformly in $|y| \leq \frac{n}{\log n}$.

It remains to show that $R_{n} \rightarrow 0$ as $n \rightarrow \infty$, uniformly in $|y| \leq \frac{n}{\log n}$. Observe that the same computation from (3.25) to (3.26) gives that

$$
R_{n}=\left(\mathrm{m}^{-(\alpha+1)}+o(1)\right) \mathbf{E}\left[1_{\left\{\tau_{\zeta} \leq n-1\right\} \cap E_{n}^{(n)}} \int_{0}^{n / \log (n)} G\left(x, x+y-S_{n-1}\right) \mathrm{d} x\right] .
$$

By (3.21), $\int_{0}^{\infty} \sup _{z \in \mathbb{R}}|G(x, z)| \mathrm{d} x \leq \frac{K}{b-1}$. Then for some constant $K^{\prime}>0$,

$$
\left|R_{n}\right| \leq K^{\prime} \mathbf{P}\left(\tau_{\zeta} \leq n-1\right)
$$

Finally, $\mathbf{P}\left(\tau_{\zeta} \leq n-1\right) \leq(n-1) \mathbf{P}\left(X_{1}<-\zeta\right) \leq n \zeta^{-\alpha} \ell(\zeta)$ which tends to 0 uniformly in $\zeta \in\left[\frac{\zeta_{n}}{4}, 4 \zeta_{n}\right]$. This yields the desired conclusion.

\section{Tightness of the minimum}

The tightness of $M_{n}-\alpha_{n}$ holds in a general setting, for instance, (1.1) and (1.4) are enough to get the upper bound of $\mathbb{P}\left(M_{n} \leq \alpha_{n}-x\right)$ which will be useful in the proof of Proposition 2.1.

Proposition 4.1. Under (1.1) and (1.4), there exists some positive constant $K$ such that for all $n \geq 2$ and $x \geq 0$,

$$
\mathbb{P}\left(M_{n} \leq \alpha_{n}-x\right) \leq K \mathrm{e}^{-x},
$$

where we recall that $\alpha_{n}:=(\alpha+1) \log n-\log \ell(n)$.

We note in passing that a computation of the second moment and an application of PaleyZygmund's inequality will give the lower bound for $\mathbb{P}\left(M_{n} \leq \alpha_{n}-x\right)$ if we assume (1.5) and an additional technical hypothesis (e.g., (A.2) in the Appendix).

Before presenting the proof of Proposition 4.1, we fix some notation which will be used throughout the rest of this paper: For $|u|=n$, we write $[\varnothing, u] \equiv\left\{u_{0}:=\varnothing, u_{1}, \ldots, u_{n-1}, u_{n}=u\right\}$ the shortest path from the root $\varnothing$ to $u$ such that $\left|u_{i}\right|=i$ for any $0 \leq i \leq n$. For any $u, v \in \mathbb{T}$, we use the partial order $u<v$ if $u$ is an ancestor of $v$ and $u \leq v$ if $u<v$ or $u=v$. By the standard words-representation in a tree, $u<v$ if and only if the word $v$ is a concatenation of the word $u$ with some word $s$, namely $v=u s$ with $|s| \geq 1$. Denote by $\mathbb{T}^{(u)}:=\{v: u \leq v\}$ the subtree rooted at $u$ and by $\mathbb{T}_{n}:=\{v:|v|=n\}$ the set of vertices at generation $n$ for any integer $n$. Let $\overleftarrow{v}$ be the parent of $v$ for any $v \neq \varnothing$. 
The following so-called many-to-one formula (4.2) can be obtained as a consequence of the spinal decomposition (see Proposition 2.3): Under (1.1), for any $n \geq 1$ and any measurable function $g: \mathbb{R}^{n} \rightarrow[0,+\infty)$,

$$
\mathbb{E}\left[\sum_{|u|=n} g\left(V\left(u_{1}\right), \ldots, V\left(u_{n}\right)\right)\right]=\mathbf{E}\left[\mathrm{e}^{S_{n}} g\left(S_{1}, \ldots, S_{n}\right)\right] .
$$

The proof of Proposition 4.1 will be based on the forthcoming three lemmas. The first one is a well-known fact in the studies of branching random walk.

Lemma 4.2. Assume (1.1). We have that

$$
\mathbb{P}(\exists u \in \mathbb{T}, V(u) \leq-x) \leq \mathrm{e}^{-x} \quad \forall x>0 .
$$

Proof. We give the proof for the sake of completeness: By considering the first generation $k \geq 1$ for which there exists some $|u|=k$ such that $V(u) \leq-x$, we get that

$$
\begin{aligned}
\mathbb{P}(\exists u \in \mathbb{T}, V(u) \leq-x) & \leq \sum_{k=1}^{\infty} \mathbb{E}\left[\sum_{|u|=k} 1_{\left\{V(u) \leq-x, V\left(u_{i}\right)>-x, \forall i \leq k\right\}}\right] \\
& =\sum_{k=1}^{\infty} \mathbf{E}\left[\mathrm{e}^{S_{k}} 1_{\left\{S_{k} \leq-x, S_{i}>-x, \forall i \leq k\right\}}\right] \\
& \leq \mathrm{e}^{-x} \sum_{k=1}^{\infty} \mathbf{P}\left(S_{k} \leq-x, S_{i}>-x, \forall i \leq k\right) \\
& \leq \mathrm{e}^{-x},
\end{aligned}
$$

where the above equality follows from the many-to-one formula (4.2).

To state the second lemma, we need to introduce some notation similar to that in (3.3) and (3.4): Recall that $\zeta_{n}:=\frac{n}{(\log n)^{3}}$. For any $u \in \mathbb{T}$, let $\tau_{\zeta_{n}}^{(u)}$ and $\tau_{\zeta_{n}}^{(2, u)}$ be the first and the second time of a large drop in the path $\left\{V\left(u_{i}\right), 1 \leq i \leq|u|\right\}$ :

$$
\begin{aligned}
\tau_{\zeta_{n}}^{(u)} & :=\inf \left\{i \in[1,|u|]: V\left(u_{i}\right)-V\left(u_{i-1}\right)<-\zeta_{n}\right\}, \\
\tau_{\zeta_{n}}^{(2, u)} & :=\inf \left\{i \in\left(\tau_{\zeta_{n}}^{(u)},|u|\right]: V\left(u_{i}\right)-V\left(u_{i-1}\right)<-\zeta_{n}\right\},
\end{aligned}
$$

with inf $\varnothing:=\infty$. Recall that $\alpha_{n}=(\alpha+1) \log n-\log \ell(n)$. Our second lemma says that for those $u$ such that $V(u) \leq \alpha_{n}-x$, necessarily there is a unique large drop before $|u|$.

Lemma 4.3. Assume (1.1) and (1.4). For any $\varepsilon>0$, there exists $n_{0}(\varepsilon)>0$ such that for any $n \geq n_{0}(\varepsilon)$ and all $x \geq 0$,

$$
\mathbb{P}\left(\exists u \in \mathbb{T}_{n}, V(u) \leq \alpha_{n}-x, \tau_{\zeta_{n}}^{(u)}>n\right) \leq \varepsilon \mathrm{e}^{-x},
$$




$$
\mathbb{P}\left(\exists u \in \mathbb{T}_{n}, V(u) \leq \alpha_{n}-x, \min _{\tau_{\zeta n}^{(u)} \leq j \leq n} V\left(u_{j}\right) \geq-x-\alpha_{n}, \tau_{\zeta_{n}}^{(2, u)} \leq n\right) \leq \varepsilon \mathrm{e}^{-x} .
$$

Consequently, for any $x>0$,

$$
\mathbb{P}\left(\exists u \in \mathbb{T}_{n}, V(u) \leq \alpha_{n}-x, \tau_{\zeta_{n}}^{(2, u)} \leq n\right) \leq \varepsilon \mathrm{e}^{-x} .
$$

We may replace in (4.7) $\min _{\tau_{\zeta n}^{(u)} \leq j \leq n} V\left(u_{j}\right) \geq-x-\alpha_{n}$ by $\min _{\tau_{\zeta n}^{(u)} \leq j \leq n} V\left(u_{j}\right) \geq-x-n^{b}$ with any constant $b \in\left(0, \alpha+\frac{1}{\alpha}-2\right)$.

Proof of Lemma 4.3. By the many-to-one formula (4.2) and using the notation (3.3) and (3.4), the probability term in (4.6) is less than

$$
\begin{aligned}
\mathbb{E}\left(\sum_{|u|=n} 1_{\left\{V(u) \leq \alpha_{n}-x, \tau_{\zeta n}^{(u)}>n\right\}}\right) & =\mathbf{E}\left(\mathrm{e}^{S_{n}} 1_{\left\{S_{n} \leq \alpha_{n}-x, \tau_{\zeta n}>n\right\}}\right) \\
& \leq \mathrm{e}^{-x+\alpha_{n}} \mathbf{P}\left(S_{n} \leq \alpha_{n}-x, \tau_{\zeta n}>n\right) \\
& \leq \mathrm{e}^{-x+\alpha_{n}} \mathbf{P}\left(S_{n}-\mathrm{m} n \leq \alpha_{n}-\mathrm{m} n, \min _{1 \leq i \leq n} X_{i} \geq-\zeta_{n}\right) \\
& \leq \mathrm{e}^{-(\mathrm{m} / 2)(\log n)^{3}} \mathrm{e}^{-x},
\end{aligned}
$$

for all large $n$, say $n \geq n_{1}$ and where we have used Lemma 3.1 for the last inequality. This proves (4.6).

Let us denote by $\mathbb{P}_{(4.7)}$ the probability term in (4.7). Then

$$
\begin{aligned}
\mathbb{P}_{(4.7)} & \leq \mathbb{E}\left[\sum_{|u|=n} 1_{\left\{V(u) \leq \alpha_{n}-x, \min _{\tau_{\zeta n}^{(u)} \leq j \leq n} V\left(u_{j}\right) \geq-x-\alpha_{n}, \tau_{\zeta n}^{(2, u)} \leq n\right\}}\right] \\
& =\mathbf{E}\left[\mathrm{e}^{S_{n}} 1_{\left\{S_{n} \leq \alpha_{n}-x, \min _{\tau_{\zeta n} \leq j \leq n} S_{j} \geq-x-\alpha_{n}, \tau_{\zeta n}^{(2)} \leq n\right\}}\right] \\
& \leq \sum_{k=1}^{\left\lceil 2 \alpha_{n}\right\rceil+1} \mathrm{e}^{k-x-\alpha_{n}} \mathbf{P}\left(S_{n}+\alpha_{n}+x \in[k-1, k), \min _{\tau_{\zeta n} \leq j \leq n} S_{j} \geq-x-\alpha_{n}, \tau_{\zeta_{n}}^{(2)} \leq n\right) .
\end{aligned}
$$

By applying (3.11) with $y \equiv-x-\alpha_{n}$, we get that [ $R$ is a non-decreasing function] for any $1 \leq k \leq\left\lceil 2 \alpha_{n}\right\rceil+1$,

$$
\mathbf{P}\left(S_{n}+\alpha_{n}+x \in[k-1, k), \min _{\tau_{\zeta n} \leq j \leq n} S_{j} \geq-x-\alpha_{n}, \tau_{\zeta_{n}}^{(2)} \leq n\right) \leq n^{1-1 / \alpha-2 \alpha} \ell_{3}(n) R\left(2 \alpha_{n}+2\right),
$$

which implies that

$$
\mathbb{P}_{(4.7)} \leq \mathrm{e}^{-x-\alpha_{n}} \mathrm{e}^{2 \alpha_{n}+1} n^{1-1 / \alpha-2 \alpha} \ell_{3}(n) R\left(2 \alpha_{n}+2\right)=\mathrm{e}^{-x} n^{2-\alpha-1 / \alpha} \ell_{4}(n)
$$

with some slowly varying function $\ell_{4}$. Since for $\alpha>1,2-\alpha-1 / \alpha<0$, (4.7) follows. 
Finally, we deduce from (4.7) and Lemma 4.2 that the probability term in (4.8) is less than $\varepsilon \mathrm{e}^{-x}+\mathbf{P}\left(\exists u \in \mathbb{T}: V(u)<-x-\alpha_{n}\right) \leq \varepsilon \mathrm{e}^{-x}+\mathrm{e}^{-\alpha_{n}-x} \leq 2 \varepsilon \mathrm{e}^{-x}$ yielding (4.7).

Below is the third and the last lemma that we need in the proof of Proposition 4.1.

Lemma 4.4. Assume (1.1) and (1.4). There exist $K, c_{4}>0$ such that for any $n$ and $L_{0} \in \mathbb{N}^{*}$ large enough, and for any $x \geq 0$ and $L \in\left[L_{0},(2+\alpha) \log n\right]$,

$$
\begin{aligned}
& \mathbb{P}\left(\exists u \in \mathbb{T}_{n}, V(u) \leq \alpha_{n}-x, \min _{\tau_{\zeta n}^{(u)} \leq j \leq n} V\left(u_{j}\right)-\left(\alpha_{n}-x\right) \in[-L,-L+1], \tau_{\zeta_{n}}^{(u)} \leq n<\tau_{\zeta_{n}}^{(2, u)}\right) \\
& \quad \leq K \mathrm{e}^{-c_{4} L} \mathrm{e}^{-x} .
\end{aligned}
$$

Consequently, there exists some constant $c_{2}>0$ such that for any $L \geq L_{0}$,

$$
\begin{aligned}
& \mathbb{P}\left(\exists u \in \mathbb{T}_{n}, V(u) \leq \alpha_{n}-x, \min _{\tau_{\zeta n}^{(u)} \leq j \leq n} V\left(u_{j}\right) \leq \alpha_{n}-x-L, \tau_{\zeta_{n}}^{(u)} \leq n<\tau_{\zeta_{n}}^{(2, u)}\right) \\
& \quad \leq K \mathrm{e}^{-c_{2} L} \mathrm{e}^{-x} .
\end{aligned}
$$

Proof. Let $\mathbb{P}_{(4.9)}$ the probability term in (4.9). Pick a constant $\beta \in\left(0, \frac{1}{4(2+\alpha)}\right)$. Notice that $L<$ $(2+\alpha) \log n$ implies $\mathrm{e}^{\beta L} \leq n^{1 / 4}$. For notational simplification, we write in this proof

$$
y \equiv y(n, x, L):=\alpha_{n}-x-L
$$

(notice that $y<\mathrm{m} n / 2$ if $n$ is large enough).

For any $u \in \mathbb{T}_{n}$ satisfying the condition in the probability term in (4.9), there exists $p \in$ $\left[\tau_{\zeta_{n}}^{(u)}, n\right]$ such that $V\left(u_{p}\right) \in[y, y+1]$. Then $\tau_{\zeta_{n}}^{(u)}=\tau_{\zeta_{n}}^{\left(u_{p}\right)}$, and

$$
\begin{aligned}
\mathbb{P}_{(4.9)} \leq & \sum_{p=1}^{n} \mathbb{P}\left(\exists u \in \mathbb{T}_{n}, \min _{\tau_{\zeta n}^{(u)} \leq j \leq n} V\left(u_{j}\right) \geq y, V\left(u_{p}\right)-y \in[0,1],\right. \\
& \left.V(u) \leq y+L, \tau_{\zeta_{n}}^{(u)} \leq p, \tau_{\zeta_{n}}^{(2, u)}>n\right) \\
\leq & \sum_{p=1}^{n-\left\lfloor\mathrm{e}^{\beta L}\right\rfloor} A_{(4.11)}(p)+\sum_{p=n-\left\lfloor\mathrm{e}^{\beta L}\right\rfloor}^{n} B_{(4.11)}(p),
\end{aligned}
$$

with

$$
\begin{aligned}
& A_{(4.11)}(p):=\mathbb{E}\left[\sum_{|u|=n} 1_{\left\{\min _{\tau_{\zeta n}(u) \leq j \leq n} V\left(u_{j}\right) \geq y, V\left(u_{p}\right)-y \in[0,1], V(u) \leq y+L, \tau_{\zeta n}^{(u)} \leq p, \tau_{\zeta n}^{(2, u)}>n\right\}}\right], \\
& B_{(4.11)}(p):=\mathbb{E}\left[\sum_{|v|=p} 1_{\left.\left\{\min _{\tau_{\zeta n}(v) \leq j \leq p} V\left(v_{j}\right) \geq y, V(v)-y \in[0,1], \tau_{\zeta n}^{(v)} \leq p<\tau_{\zeta n}^{(2, v)}\right\}\right]}\right.
\end{aligned}
$$


where the sum of the expectation term of $B_{(4.11)}(p)$ is obtained by considering $v=u_{p}$ satisfying $V\left(u_{p}\right) \in[y, y+1]$. We omitted the dependence on $n$ in both $A_{(4.11)}(p)$ and $B_{(4.11)}(p)$. By using (4.2), we have

$$
\begin{aligned}
B_{(4.11)}(p) & =\mathbf{E}\left[\mathrm{e}^{S_{p}} 1_{\left\{\min _{\tau_{\zeta n} \leq j \leq p} S_{j} \geq y, S_{p}-y \in[0,1], \tau_{\zeta n} \leq p<\tau_{\zeta n}^{(2)}\right\}}\right] \\
& \leq \mathrm{e}^{y+1} \mathbf{P}\left(\min _{\tau_{\zeta n} \leq j \leq p} S_{j} \geq y, S_{p}-y \in[0,1], \tau_{\zeta_{n}} \leq p<\tau_{\zeta_{n}}^{(2)}\right) \\
& \leq K^{\prime} \mathrm{e}^{y} \mathrm{e}^{-\alpha_{p}},
\end{aligned}
$$

where the last inequality follows from (3.12) by remarking that $\frac{\zeta_{p}}{4} \leq \zeta_{n} \leq 4 \zeta_{p}$ for any $p \in$ $\left[n-\left\lfloor\mathrm{e}^{\beta L}\right\rfloor, n\right] ;$ moreover $\mathrm{e}^{-\alpha_{p}} \sim \mathrm{e}^{-\alpha_{n}}$, so for all large $n$,

$$
\sum_{p=n-\left\lfloor\mathrm{e}^{\beta L}\right\rfloor}^{n} B_{(4.11)}(p) \leq 2 K^{\prime} \mathrm{e}^{y+\beta L} \mathrm{e}^{-\alpha_{n}} \leq K \mathrm{e}^{-x-L / 2} .
$$

It remains to estimate $A_{(4.11)}(p)$. By applying (4.2),

$$
\begin{aligned}
A_{(4.11)}(p) & =\mathbf{E}\left[\mathrm{e}^{S_{n}} 1_{\left\{\min _{\tau_{\zeta n} \leq j \leq n} S_{j} \geq y, S_{p}-y \in[0,1], S_{n} \leq y+L, \tau_{\zeta n} \leq p, \tau_{\zeta n}^{(2)}>n\right\}}\right] \\
& \leq \mathrm{e}^{y+L} \mathbf{P}\left(\min _{\tau_{\zeta n} \leq j \leq n} S_{j} \geq y, S_{p}-y \in[0,1], S_{n}-y \in[0, L], \tau_{\zeta_{n}} \leq p, \tau_{\zeta_{n}}^{(2)}>n\right) .
\end{aligned}
$$

By applying the Markov property at time $p$, we see that the above probability term is equal to

$$
\mathbf{E}\left[1_{\left\{\min _{\tau_{\zeta n} \leq j \leq p} S_{j} \geq y, S_{p}-y \in[0,1], \tau_{\zeta n} \leq p<\tau_{\zeta n}^{(2)}\right\}} \mathbf{P}_{S_{p}}\left(\underline{S}_{n-p} \geq y, S_{n-p}-y \in[0, L], \tau_{\zeta_{n}}>n-p\right)\right] .
$$

For any $z \equiv S_{p} \in[y, y+1], \mathbf{P}_{z}\left(\underline{S}_{n-p} \geq y, S_{n-p}-y \in[0, L], \tau_{\zeta_{n}}>n-p\right) \leq \mathbf{P}\left(\underline{S}_{n-p} \geq\right.$ $\left.-1, S_{n-p} \in[-1, L+1], \tau_{\zeta_{n}}>n-p\right)$. Recalling that $y=\alpha_{n}-x-L$, we get

$$
A_{(4.11)}(p) \leq \mathrm{e}^{\alpha_{n}-x} I_{(4.13)} J_{(4.13)}
$$

with

$$
\begin{aligned}
I_{(4.13)} & :=\mathbf{P}\left(\min _{\tau_{\zeta_{n}} \leq j \leq p} S_{j} \geq y, S_{p}-y \in[0,1], \tau_{\zeta_{n}} \leq p<\tau_{\zeta_{n}}^{(2)}\right), \\
J_{(4.13)} & :=\mathbf{P}\left(\underline{S}_{n-p} \geq-1, S_{n-p} \in[-1, L+1], \tau_{\zeta_{n}}>n-p\right) .
\end{aligned}
$$

For $1 \leq p<\left\lfloor\frac{n}{4}\right\rfloor$, we apply Lemma 3.1 to see that $J_{(4.13)} \leq \mathbf{P}\left(S_{n-p} \leq L+1, \tau_{\zeta_{n}}>n-p\right) \leq$ $K \mathrm{e}^{-(3 \mathrm{~m} n / 4-(L+1)) / \zeta_{n}}$, hence for $1 \leq p<\left\lfloor\frac{n}{4}\right\rfloor$,

$$
A_{(4.11)}(p) \leq K \mathrm{e}^{\alpha_{n}-x} \mathrm{e}^{-(3 \mathrm{~m} n / 4-(L+1)) / \zeta_{n}} \leq \mathrm{e}^{-(\mathrm{m} / 2)(\log n)^{3}} \mathrm{e}^{-x} .
$$

For $\left\lfloor\frac{n}{4}\right\rfloor \leq p \leq n-\left\lfloor\mathrm{e}^{\beta L}\right\rfloor$, we apply (3.12) for $I_{(4.13)}$ (with $y \equiv \alpha_{n}-L-x \leq \alpha_{n} \leq \frac{\mathrm{m}}{2} p$ and $\left.\zeta=\zeta_{n} \in\left[\frac{\zeta_{p}}{4}, 4 \zeta_{p}\right]\right)$, and $L+1$ times Lemma 3.4 for $J_{(4.13)}$ (recall that $n-p \geq\left\lfloor\mathrm{e}^{\beta L}\right\rfloor \geq \mathrm{e}^{\beta L_{0}}$ 
large and thus $\left.L \leq \frac{m}{2}(n-p)\right)$ we get

$$
A_{(4.11)}(p) \leq K^{\prime} \mathrm{e}^{\alpha_{n}-x} \mathrm{e}^{-\alpha_{n}}(L+1) \frac{\ell(n-p)}{(n-p)^{\alpha}} \leq K^{\prime \prime} \mathrm{e}^{-x}(L+1) \frac{\ell(n-p)}{(n-p)^{\alpha}},
$$

which together with (4.14) yield that

$$
\begin{aligned}
\sum_{p=1}^{n-\left\lfloor\mathrm{e}^{\beta L}\right\rfloor} A_{(4.11)}(p) & \leq \mathrm{e}^{-x} n \mathrm{e}^{-(\mathrm{m} / 2)(\log n)^{3}}+K^{\prime \prime} \mathrm{e}^{-x}(L+1) \sum_{p=\lfloor n / 4\rfloor}^{n-\left\lfloor\mathrm{e}^{\beta L}\right\rfloor} \frac{\ell(n-p)}{(n-p)^{\alpha}} \\
& \leq K \mathrm{e}^{-x} \mathrm{e}^{-((\alpha-1) / 2) \beta L},
\end{aligned}
$$

proving (4.9).

It remains to prove (4.10). Let $c_{3}:=\frac{1}{2(\alpha+2)}$. Remark that if $L \geq \frac{\alpha_{n}}{1-c_{3}}$, then $\alpha_{n}-L \leq-c_{3} L$ and it follows from Lemma 4.2 that

$$
\mathbb{P}\left(\exists u \in \mathbb{T}_{n}, \min _{\tau_{\zeta n}^{(u)} \leq j \leq n} V\left(u_{j}\right) \leq \alpha_{n}-x-L, \tau_{\zeta_{n}}^{(u)} \leq n\right) \leq \mathrm{e}^{-c_{3} L-x} .
$$

Therefore, it is enough to treat the case $L_{0} \leq L<\frac{\alpha_{n}}{1-c_{3}}$. As $n \geq n_{0}, \frac{\alpha_{n}}{1-c_{3}} \leq(2+\alpha) \log n$. Then the probability term in (4.10) is less than

$$
\begin{aligned}
& \mathbb{P}\left(\exists u \in \mathbb{T}_{n}, V(u) \leq \alpha_{n}-x, \min _{\tau_{\zeta n}^{(u)} \leq j \leq n} V\left(u_{j}\right)-\left(\alpha_{n}-x\right) \leq-c_{3} L, \tau_{\zeta_{n}}^{(u)} \leq n<\tau_{\zeta_{n}}^{(2, u)}\right) \\
& \quad+\sum_{k=L}^{\alpha_{n}+c_{3} L} \mathbb{P}\left(\exists u \in \mathbb{T}_{n}, V(u) \leq \alpha_{n}-x, \min _{\tau_{\zeta n}^{(u)} \leq j \leq n} V\left(u_{j}\right)-\left(\alpha_{n}-x\right) \in[-k,-k+1)\right. \\
& \left.\quad \tau_{\zeta_{n}}^{(u)} \leq n<\tau_{\zeta_{n}}^{(2, u)}\right) \\
& \quad \leq \mathrm{e}^{-c_{3} L-x}+\sum_{k=L}^{\alpha_{n} /\left(1-c_{3}\right)} \mathrm{e}^{-c_{4} k-x} \\
& \quad \leq \mathrm{e}^{-c_{3} L-x}+K^{\prime} \mathrm{e}^{-c_{4} L-x} .
\end{aligned}
$$

We get (4.10) by choosing $c_{2}:=\min \left(c_{3}, c_{4}\right)$.

Now we can tackle the proof of Proposition 4.1.

Proof of Proposition 4.1. Fix an arbitrary integer $L \geq L_{0}\left(L_{0}\right.$ as in Lemma 4.4) and consider $n$ large enough to have $L \in\left[L_{0},(2+\alpha) \log n\right]$. Then

$$
\mathbb{P}\left(M_{n} \leq \alpha_{n}-x\right) \leq \mathbb{P}_{(4.15)}^{(1)}+\mathbb{P}_{(4.15)}^{(2)}+\mathbb{P}_{(4.15)}^{(3)}+\mathbb{P}_{(4.15)}^{(4)},
$$


with

$$
\begin{aligned}
\mathbb{P}_{(4.15)}^{(1)}:= & \mathbb{P}(\exists u \in \mathbb{T}, V(u) \leq-x), \\
\mathbb{P}_{(4.15)}^{(2)}:= & \mathbb{P}\left(\exists u \in \mathbb{T}_{n}, V(u) \leq \alpha_{n}-x, \min _{\tau_{\zeta n}^{(u)} \leq j \leq n} V\left(u_{j}\right)>-x, \tau_{\zeta_{n}}^{(2, u)} \leq n\right) \\
& +\mathbb{P}\left(\exists u \in \mathbb{T}_{n}, V(u) \leq \alpha_{n}-x, \tau_{\zeta n}^{(u)}>n\right), \\
\mathbb{P}_{(4.15)}^{(3)}:= & \sum_{k=L}^{\alpha_{n}+1} \mathbb{P}\left(\exists u \in \mathbb{T}_{n}, V(u) \leq \alpha_{n}-x, \min _{\tau_{\zeta n}^{(u)} \leq j \leq n} V\left(u_{j}\right)-\left(\alpha_{n}-x\right) \in[-k,-k+1),\right. \\
& \left.\tau_{\zeta_{n}}^{(u)} \leq n<\tau_{\zeta_{n}}^{(2, u)}\right), \\
\mathbb{P}_{(4.15)}^{(4)}:= & \mathbb{P}\left(\exists u \in \mathbb{T}_{n}, V(u) \leq \alpha_{n}-x, \min _{\tau_{\zeta n}^{(u)} \leq j \leq n} V\left(u_{j}\right) \geq \alpha_{n}-x-L, \tau_{\zeta_{n}}^{(u)} \leq n<\tau_{\zeta_{n}}^{(2, u)}\right) .
\end{aligned}
$$

Based on (4.3), (4.6), (4.8) and (4.9), we only need to estimate $\mathbb{P}_{(4.15)}^{(4)}$. By the many-to-one formula (4.2), we get that

$$
\begin{aligned}
\mathbb{P}_{(4.15)}^{(4)} & \leq \mathbb{E}\left[\sum_{|u|=n} 1_{\left\{V(u) \leq \alpha_{n}-x, \min _{\tau_{\zeta n}^{(u)} \leq j \leq n} V\left(u_{j}\right) \geq \alpha_{n}-x-L, \tau_{\zeta n}^{(u)} \leq n<\tau_{\zeta n}^{(2, u)}\right\}}\right] \\
& \leq \mathbf{E}\left[\mathrm{e}^{S_{n}} 1_{\left\{S_{n} \leq \alpha_{n}-x, \min _{\tau_{\zeta n} \leq j \leq n} S_{j} \geq \alpha_{n}-x-L, \tau_{\zeta n} \leq n<\tau_{\zeta n}^{(2)}\right\}}\right] \\
& \leq \mathrm{e}^{\alpha_{n}-x} \mathbf{P}\left(S_{n} \leq \alpha_{n}-x, \min _{\tau_{\zeta n} \leq j \leq n} S_{j} \geq \alpha_{n}-x-L, \tau_{\zeta_{n}} \leq n<\tau_{\zeta_{n}}^{(2)}\right) \\
& \leq \mathrm{e}^{\alpha_{n}-x} \sum_{k=1}^{L} \mathbf{P}\left(S_{n}-\alpha_{n}+x \in[-k,-k+1], \min _{\tau_{\zeta n} \leq j \leq n} S_{j} \geq \alpha_{n}-x-L, \tau_{\zeta_{n}} \leq n<\tau_{\zeta_{n}}^{(2)}\right) \\
& \leq K \mathrm{e}^{-x} L^{2},
\end{aligned}
$$

where to obtain the last estimate, we have used the display (3.12) (with $y=-\alpha_{n}+x+L$, $a=L-k$ there) and the fact that $R(a+1) \sim \mathrm{m} a$ as $a \rightarrow \infty$. This completes the proof of Proposition 4.1.

We end this section by a lemma which will be used in Section 5 .

Lemma 4.5. Assume (1.1) and (1.4). Let $\varepsilon>0$. For any $L>0$, there are some integers $T=$ $T(\varepsilon, L)$ and $n_{0}=n_{0}(\varepsilon, L)>T$ such that for all $n \geq n_{0}$ and all $x>0$ :

$$
\begin{aligned}
& \mathbb{P}\left(\exists u \in \mathbb{T}_{n}, V(u) \leq \alpha_{n}-x, \min _{\tau_{\zeta n}^{(u)} \leq j \leq n} V\left(u_{j}\right) \geq \alpha_{n}-x-L, \tau_{\zeta_{n}}^{(u)} \leq n-T, \tau_{\zeta_{n}}^{(2, u)}>n\right) \\
& \quad \leq \varepsilon \mathrm{e}^{-x} .
\end{aligned}
$$


Proof. Denote by $\mathbb{P}_{(4.16)}$ the probability term in (4.16) and write $y=\alpha_{n}-x-L$. Then by the many-to-one formula

$$
\begin{aligned}
\mathbb{P}_{(4.16)} & \leq \mathbb{E}\left[\sum_{|u|=n} 1_{\left\{V(u) \leq y+L, \min _{\tau_{\zeta n}^{(u)} \leq j \leq n} V\left(u_{j}\right) \geq y, \tau_{\zeta n}^{(u)} \leq n-T, \tau_{\zeta n}^{(2, u)}>n\right\}}\right] \\
& =\mathbf{E}\left[\mathrm{e}^{S_{n}} 1_{\left\{S_{n} \leq y+L, \min _{\tau_{\zeta n} \leq j \leq n} S_{j} \geq y, \tau_{\zeta n} \leq n-T, \tau_{\zeta n}^{(2)}>n\right\}}\right] \\
& \leq \sum_{i=1}^{n-T} \sum_{k=1}^{\lfloor L\rfloor+1} \mathrm{e}^{y+k} \mathbf{P}\left(S_{n}-y \in[k-1, k), \min _{\tau_{\zeta n} \leq j \leq n} S_{j} \geq y, \tau_{\zeta_{n}}=i, \tau_{\zeta_{n}}^{(2)}>n\right) .
\end{aligned}
$$

Each probability term in the above double sum is less than, by (3.15), $K \mathbf{P}\left(\bar{S}_{n-i+1} \leq k\right) \mathrm{e}^{-\alpha_{n}}+$ $n^{-(\alpha+\gamma-1)} \ell_{3}(n) \leq K \mathbf{P}\left(\bar{S}_{n-i+1} \leq L+1\right) \mathrm{e}^{-\alpha_{n}}+n^{-(\alpha+\gamma-1)} \ell_{3}(n)$, hence by taking the double sum over $i$ and $k$,

$$
\mathbb{P}_{(4.16)} \leq K^{\prime} \mathrm{e}^{-x} \sum_{j=T}^{n} \mathbf{P}\left(\bar{S}_{j} \leq L+1\right)+\mathrm{e}^{-x} \mathrm{e}^{\alpha_{n}} n^{-(\alpha+\gamma-2)} \ell_{3}(n) .
$$

Taking $T=T(\varepsilon, L)$ large enough such that $\sum_{j=T}^{\infty} \mathbf{P}\left(\bar{S}_{j} \leq L+1\right)<\frac{\varepsilon}{2 K^{\prime}}$ (according to Lemma 3.2) and $n_{0}$ large enough so that $\mathrm{e}^{\alpha_{n}} n^{-(\alpha+\gamma-2)} \ell_{3}(n) \leq \frac{\varepsilon}{2}$ for all $n \geq n_{0}$, we get (4.16).

\section{Proof of Proposition 2.1}

The proof of Proposition 2.1 relies on the analysis of the trajectory of a particle which reaches the minimum at time $n$. The main step in the proof is to show that in the i.i.d. case, all minimal particles will have a (unique) common large drop; see Proposition 5.1 for the precise statement.

At first, we shall make use of the estimates in Section 4 to localize the generation at which the minimal particles make a large drop.

Let $\varepsilon>0$ be small and $x>0$. Let $L \equiv L(\varepsilon) \geq L_{0}$ where $L_{0}$ is given by Lemma 4.4 be such that $\mathrm{Ke}^{-c_{2} L}<\varepsilon$. Consider the event that there is some $u \in \mathbb{T}_{n}$ such that $V(u) \leq \alpha_{n}-x$. By (4.6) and (4.8), with a cost at most $2 \varepsilon \mathrm{e}^{-x}$, we may assume that $\tau_{\zeta_{n}}^{(u)} \leq n$, which in view of (4.10) yields that we may furthermore assume $\min _{\tau_{\zeta n}^{(u)} \leq j \leq n} V\left(u_{j}\right)>\alpha_{n}-x-L$ with an extra cost at most equal to $\varepsilon \mathrm{e}^{-x}$. Finally by (4.16), there exists some integer $T \equiv T(L, \varepsilon)$ such that we may assume $\tau_{\zeta_{n}}^{(u)}>n-T$ with an extra cost at most equal to $\varepsilon \mathrm{e}^{-x}$. Consequently, for all large $n \geq n_{1}(\varepsilon)$ and for all $x>0$,

$$
\begin{aligned}
\mathbb{P} & \left(M_{n} \leq \alpha_{n}-x\right) \\
& =\mathbb{E}\left[\frac{\sum_{|u|=n} 1_{\left\{M_{n}=V(u) \leq \alpha_{n}-x, \min _{\tau_{\zeta n}^{(u)} \leq j \leq n} V\left(u_{j}\right) \geq \alpha_{n}-x-L, \tau_{\zeta n}^{(u)} \in[n-T, n]\right\}}}{\sum_{|u|=n} 1_{\left\{V(u)=M_{n}\right\}}}\right]+O(\varepsilon) \mathrm{e}^{-x} \\
& =\mathbb{E}_{\mathbb{Q}}\left[\mathrm{e}^{V\left(w_{n}\right)} \frac{1_{\left\{M_{n}=V\left(w_{n}\right) \leq \alpha_{n}-x, \min _{\tau_{\zeta n}^{\left(w_{n}\right)} \leq j \leq n} V\left(w_{j}\right) \geq \alpha_{n}-x-L, \tau_{\zeta n}^{\left(w_{n}\right)} \in[n-T, n]\right\}}}{\sum_{|u|=n} 1_{\left\{V(u)=M_{n}\right\}}}\right]+O(\varepsilon) \mathrm{e}^{-x},
\end{aligned}
$$


where we have used the change of measure (cf. Proposition 2.3) for the last equality and $O(\varepsilon)$ denotes, as usual, some term bounded by a numerical constant times $\varepsilon$ (here by $5 \varepsilon$ ).

The next goal is to analyze the number of minima $\sum_{|u|=n} 1_{\left\{V(u)=M_{n}\right\}}$ and the set $\left\{M_{n}=\right.$ $\left.V\left(w_{n}\right)\right\}$ in (5.1). To this end, we consider the following event:

$$
\mathcal{E}_{n}(x):=\left\{\forall k \leq \tau_{\zeta_{n}}^{\left(w_{n}\right)}, \forall v \in \mathbb{B}\left(w_{k}\right), \min _{u \in \mathbb{T}^{(v)},|u|=n} V(u)>\alpha_{n}-x\right\},
$$

where $\mathbb{B}\left(w_{k}\right)$, defined in (1.9), denotes the set of brothers of $w_{k}$, and $\mathbb{T}^{(v)}$ denotes as before the subtree rooted at $v$. The following result, the key in the proof of Proposition 2.1, ensures that in the i.i.d. case, all minimal particles must be descendants of $w_{\ell}$ if $\ell:=\tau_{\zeta_{n}}^{\left(w_{n}\right)}$ is the first large drop time of the path $\left(V\left(w_{i}\right)_{0 \leq i \leq n}\right)$.

Proposition 5.1 (i.i.d. case). Assume (1.1), (1.4) and (1.5). Suppose that $\mathscr{L}=\sum_{i=1}^{v} \delta_{\left\{\xi_{i}\right\}}$ with $\left(\xi_{i}\right)$ i.i.d. and independent of $v$. Then for any $\varepsilon, L, T>0$ there exists $x_{1}>0$ such that for any $n$ large enough and $x \geq x_{1}$,

$$
\begin{aligned}
& \mathbb{Q}\left(V\left(w_{n}\right) \leq \alpha_{n}-x, \min _{\tau_{\zeta n}^{\left(w_{n}\right)} \leq j \leq n} V\left(w_{j}\right) \geq \alpha_{n}-x-L, \tau_{\zeta_{n}}^{\left(w_{n}\right)} \in[n-T, n],\left(\mathcal{E}_{n}(x)\right)^{c}\right) \\
& \quad \leq \varepsilon \mathrm{e}^{-\alpha_{n}} .
\end{aligned}
$$

Consequently, for all $x \geq x_{1}$ and all large $n$,

$$
\mathbb{E}_{\mathbb{Q}}\left[\mathrm{e}^{V\left(w_{n}\right)} 1_{\left\{V\left(w_{n}\right) \leq \alpha_{n}-x, \min _{\tau_{\zeta n}^{\left(w_{n}\right)} \leq j \leq n} V\left(w_{j}\right) \geq \alpha_{n}-x-L, \tau_{\zeta n}^{\left(w_{n}\right)} \in[n-T, n],\left(\mathcal{E}_{n}(x)\right)^{c}\right\}}\right] \leq \varepsilon \mathrm{e}^{-x} .
$$

Remark 5.2. Without the i.i.d. assumption on $\mathscr{L}$, Proposition 5.1 still holds if we change the definition of $\mathcal{E}_{n}(x)$ by replacing $k \leq \tau_{\zeta_{n}}^{\left(w_{n}\right)}$ by $k<\tau_{\zeta_{n}}^{\left(w_{n}\right)}$.

Let us postpone the proof of Proposition 5.1 to Section 6 and give the proof of Proposition 2.1.

Proof of Proposition 2.1. It follows from (5.1) and (5.4) that for any $x \geq x_{1}$, for $n$ large enough,

$$
\begin{aligned}
\mathbb{P} & \left(M_{n} \leq \alpha_{n}-x\right) \\
& =\mathbb{E}_{\mathbb{Q}}\left[\mathrm{e}^{V\left(w_{n}\right)} \frac{1_{\left\{M_{n}=V\left(w_{n}\right) \leq \alpha_{n}-x, \min _{\tau_{\zeta n}^{\left(w_{n}\right)} \leq j \leq n} V\left(w_{j}\right) \geq \alpha_{n}-x-L, \tau_{\zeta n}^{\left(w_{n}\right)} \in[n-T, n]\right\}}}{\sum_{|u|=n} 1_{\left\{V(u)=M_{n}\right\}}}, \mathcal{E}_{n}(x)\right]+O(\varepsilon) \mathrm{e}^{-x} \\
& =\sum_{k=n-T}^{n} \mathbb{E}_{\mathbb{Q}}\left[\mathrm{e}^{V\left(w_{n}\right)} \frac{1_{\left\{M_{n}=V\left(w_{n}\right) \leq \alpha_{n}-x, \min _{k \leq j \leq n} V\left(w_{j}\right) \geq \alpha_{n}-x-L, \tau_{\zeta n}^{\left(w_{n}\right)}=k\right\}}}{\sum_{|u|=n, u \in \mathbb{T}^{\left(w_{k}\right)}} 1_{\left\{V(u)=V\left(w_{n}\right)\right\}}}, \mathcal{E}_{n}(x)\right]+O(\varepsilon) \mathrm{e}^{-x} \\
& =\sum_{k=n-T}^{n} \mathbb{E}_{\mathbb{Q}}\left[A_{(5.5)}(k)\right]+O(\varepsilon) \mathrm{e}^{-x},
\end{aligned}
$$


where

$$
A_{(5.5)}(k):=\mathrm{e}^{V\left(w_{n}\right)} \frac{1_{\left\{M_{n}=V\left(w_{n}\right) \leq \alpha_{n}-x, \min _{k \leq j \leq n} V\left(w_{j}\right) \geq \alpha_{n}-x-L, \tau_{\zeta n}^{\left(w_{n}\right)}=k\right\}}}{\sum_{|u|=n, u \in \mathbb{T}^{\left(w_{k}\right)}} 1_{\left\{V(u)=V\left(w_{n}\right)\right\}}}, \quad n-T \leq k \leq n,
$$

and in the last equality in (5.5) we have again used (5.4). Obviously, the following upper bound holds:

$$
A_{(5.5)}(k) \leq \mathrm{e}^{V\left(w_{n}\right)} 1_{\left\{V\left(w_{n}\right) \leq \alpha_{n}-x, \min _{k \leq j \leq n} V\left(w_{j}\right) \geq \alpha_{n}-x-L, \tau_{\zeta n}^{\left(w_{n}\right)}=k\right\}} .
$$

Recall that under $\mathbb{Q},\left(V\left(w_{j}\right), j \geq 0\right)$ is distributed as the random walk $\left(S_{j}, j \geq 0\right)$ under $\mathbf{P}$. Then

$$
\mathbb{E}_{\mathbb{Q}}\left[A_{(5.5)}(k)\right] \leq \mathrm{e}^{\alpha_{n}-x} \mathbf{P}\left(S_{n} \leq \alpha_{n}-x, \min _{k \leq j \leq n} S_{j} \geq \alpha_{n}-x-L, \tau_{\zeta_{n}}=k\right) \leq K_{L} \mathrm{e}^{-x}
$$

by using (3.11) and (3.12).

Observe that $\left\{\tau_{\zeta_{n}}^{\left(w_{n}\right)}=k\right\}=\left\{\tau_{\zeta_{n}}^{\left(w_{k}\right)}=k\right\}$. We deduce from the Markov property at $k$ that

$$
\mathbb{E}_{\mathbb{Q}}\left[A_{(5.5)}(k)\right]=\mathrm{e}^{\alpha_{n}-x} \mathbb{E}_{\mathbb{Q}}\left[1_{\left\{\tau_{\zeta n}^{\left(w_{k}\right)}=k, V\left(w_{k}\right) \geq \alpha_{n}-x-L\right\}} F_{n-k}^{(L)}\left(V\left(w_{k}\right)-\left(\alpha_{n}-x-L\right)\right)\right],
$$

where for any $j \geq 0, F_{j}^{(L)}: \mathbb{R}_{+} \rightarrow[0,1]$ is the measurable function defined as follows:

$$
F_{j}^{(L)}(s):=\mathrm{e}^{s-L} \mathbb{E}_{\mathbb{Q}}\left[\mathrm{e}^{V\left(w_{j}\right)} \frac{1_{\left\{M_{j}=V\left(w_{j}\right)\right\}} 1_{\left\{V\left(w_{j}\right) \leq L-s, \underline{V}\left(w_{j}\right) \geq-s\right\}}}{\sum_{|v|=j} 1_{\left\{V(v)=M_{j}\right\}}}\right],
$$

where $\underline{V}\left(w_{j}\right):=\min _{0 \leq i \leq j} V\left(w_{i}\right)$. Recall that under $\mathbb{Q},\left(V\left(w_{k}\right)\right)_{k \geq 0}$ is distributed as $\left(S_{k}\right)_{k \geq 0}$ under $\mathbf{P}$. Then by combining (5.5) and (5.8), we get $\mathbb{P}\left(M_{n} \leq \alpha_{n}-x\right)=\sum_{j=0}^{T} \mathrm{e}^{\alpha_{n}-x} \mathbf{E}\left[1_{\left\{\tau_{\zeta n}=n-j, S_{n-j} \geq \alpha_{n}-x-L\right\}} F_{j}^{(L)}\left(S_{n-j}-\left(\alpha_{n}-x-L\right)\right)\right]+O(\varepsilon) \mathrm{e}^{-x}$.

Observe that $F_{j}^{(L)}(s) \leq \mathrm{e}^{s-L} \mathbb{E}_{\mathbb{Q}}\left[\mathrm{e}^{V\left(w_{j}\right)} 1_{\left\{V\left(w_{j}\right) \leq L-s, \underline{V}\left(w_{j}\right) \geq-s\right\}}\right] \leq \mathbb{Q}\left(V\left(w_{j}\right) \leq L-s\right)=$ $\mathbf{P}\left(S_{j} \leq L-s\right)$, it follows from (1.4) that for any $s \geq 0$,

$$
F_{j}^{(L)}(s) \leq \mathbf{P}\left(S_{j} \leq L-s\right) \leq j \mathbf{P}\left(X \leq \frac{L-s}{j}\right) \leq K_{j, L}(1+s)^{-\alpha} \ell(s) .
$$

In view of (5.10), we can apply Lemma 3.6 to $F_{j}^{(L)}(\cdot)$, for any fixed $0 \leq j<T$. This gives that for any $L \geq L_{1}$ and large $T$, for all large $n \geq n_{1}(\varepsilon, L, T)$ and $x \in\left[x_{1}, \frac{n}{\log n}\right]$,

$$
\left|\mathbb{P}\left(M_{n} \leq \alpha_{n}-x\right)-\mathrm{m}^{-(\alpha+1)} \mathrm{e}^{-x} \sum_{j=0}^{T} \int_{0}^{\infty} F_{j}^{(L)}(s) \mathrm{d} s\right| \leq O(\varepsilon) \mathrm{e}^{-x} .
$$


Notice that in view of (4.1), (5.11) implies that for any $L \geq L_{1}$ and large $T$,

$$
\mathrm{m}^{-(\alpha+1)} \sum_{j=0}^{T} \int_{0}^{\infty} F_{j}^{(L)}(s) \mathrm{d} s \leq K+O(\varepsilon) .
$$

Now we apply the Fubini theorem to see that

$$
\int_{0}^{\infty} F_{j}^{(L)}(s) \mathrm{d} s=\mathbb{E}_{\mathbb{Q}}\left[\mathrm{e}^{V\left(w_{j}\right)}\left(\mathrm{e}^{-V\left(w_{j}\right)}-\mathrm{e}^{-L-\underline{V}\left(w_{j}\right)}\right) \frac{1_{\left\{M_{j}=V\left(w_{j}\right)\right\}} 1_{\left\{V\left(w_{j}\right) \leq L\right\}}}{\sum_{|v|=j} 1_{\left\{V(v)=M_{j}\right\}}}\right] .
$$

On the other hand, we deduce from the monotone convergence theorem (when $T$ and $L$ tend to $\infty)$ that

$$
\begin{aligned}
\lim _{L \rightarrow \infty} \lim _{T \rightarrow \infty} \mathrm{m}^{-(\alpha+1)} \sum_{j=0}^{T} \int_{0}^{\infty} F_{j}^{(L)}(u) \mathrm{d} u & =\mathrm{m}^{-(\alpha+1)} \sum_{j=0}^{\infty} \mathbb{E}_{\mathbb{Q}}\left[\frac{1_{\left\{M_{j}=V\left(w_{j}\right)\right\}}}{\sum_{|v|=j} 1_{\left\{V(v)=M_{j}\right\}}}\right] \\
& =\mathrm{m}^{-(\alpha+1)} \sum_{j=0}^{\infty} \mathbb{E}\left[\mathrm{e}^{-M_{j}}\right] \\
& =c_{*},
\end{aligned}
$$

where in the second equality we have used the change of measure (2.3) to see that for any $j \geq 1$, $\mathbb{E}_{\mathbb{Q}}\left[\frac{1_{\left\{M_{j}=V\left(w_{j}\right)\right\}}}{\sum_{|v|=j} 1_{\left\{V(v)=M_{j}\right\}}}\right]=\mathbb{E}\left[\sum_{|u|=j} \mathrm{e}^{-V(u)} \frac{1_{\left\{M_{j}=V(u)\right\}}}{\sum_{|v|=j} 1_{\left\{V(v)=M_{j}\right\}}}\right]=\mathbb{E}\left[\mathrm{e}^{-M_{j}}\right]$. Moreover, $c_{*} \leq K$ thanks to (5.12) and the fact that $\varepsilon$ can be arbitrarily small. This and (5.11) yield Proposition 2.1.

\section{Proof of Proposition 5.1}

Recall from (1.9) that $\mathbb{B}(u)$ is the set of brothers of $u$ for any $u \in \mathbb{T} \backslash\{\varnothing\}$. To prove Proposition 5.1, we shall discuss whether the vertex $w_{n}$ is good or not: If $w_{n}$ is good, then with high probability no descendent of the particles in $\bigcup_{k \leq \tau_{\zeta n}^{\left(w_{n}\right)}} \mathbb{B}\left(w_{k}\right)$ stays at a position smaller than $\alpha_{n}-x$ (i.e., close to the minimum $M_{n}$ ). If $w_{n}$ is not good, then with high probability $w_{n}$ cannot be a minimal particle (see the forthcoming Lemma 6.1).

Let us give the precise definition of good vertices: Fix $0<\varrho<\min \left(\frac{\alpha-1}{2}, \frac{1}{12}\right)$. Let $B>0$ be a large constant and $J$ be a large integer. Recall (4.4) and (4.5).

Let us say that $u \in \mathbb{T}_{n}$ is a good vertex if for any $x \geq 0$,

$$
\tau_{\zeta_{n}}^{(2, u)}>n \geq \tau_{\zeta_{n}}^{(u)}>J \quad \text { and } \quad \sum_{v \in \mathbb{B}\left(u_{k}\right)} \mathrm{e}^{-(V(v)+x)} \leq \begin{cases}\mathrm{e}^{B-x}, & \text { if } 1 \leq k \leq J, \\ \mathrm{e}^{-k^{\varrho}}, & \text { if } J<k<\tau_{\zeta_{n}}^{(u)} .\end{cases}
$$

The condition $\left\{n \geq \tau_{\zeta_{n}}^{(u)}>J\right\}$ will be automatically satisfied in the event that we are interested in.

The following lemma treats the case when $w_{n}$ is not good. 
Lemma 6.1. Under (1.1), (1.4) and (1.5), for any $L, T, \varepsilon>0$, there exists $J(L, T, \varepsilon)$ such that for all $J \geq J(L, T, \varepsilon)$, there exists $B(J, L, T, \varepsilon)>0$ such that for all $B \geq B(J, L, T, \varepsilon)$, for any $n$ large enough and $x \geq 0$,

$$
\begin{aligned}
& \mathbb{Q}\left(V\left(w_{n}\right) \leq \alpha_{n}-x, \min _{\tau_{\zeta n}^{\left(w_{n}\right)} \leq j \leq n} V\left(w_{j}\right) \geq \alpha_{n}-x-L, \tau_{\zeta_{n}}^{\left(w_{n}\right)} \in[n-T, n], w_{n} \text { not good }\right) \\
& \quad \leq \varepsilon \mathrm{e}^{-\alpha_{n}} .
\end{aligned}
$$

We stress that we do not need the i.i.d. assumption in Lemma 6.1.

By admitting Lemma 6.1 for the moment, we can prove Proposition 5.1.

Proof of Proposition 5.1. For brevity, we use the following notation:

$$
F_{n} \equiv F_{n, T, x}:=\left\{V\left(w_{n}\right) \leq \alpha_{n}-x, \min _{\tau_{\zeta n}^{\left(w_{n}\right)} \leq i \leq n} V\left(w_{j}\right) \geq \alpha_{n}-x-L, \tau_{\zeta_{n}}^{\left(w_{n}\right)} \in[n-T, n]\right\} .
$$

By (6.2), the probability term in (5.3) is less than

$$
\begin{aligned}
& \varepsilon \mathrm{e}^{-\alpha_{n}}+\mathbb{Q}\left(F_{n}, w_{n} \operatorname{good},\left(\mathcal{E}_{n}(x)\right)^{c}\right) \\
& \leq \varepsilon \mathrm{e}^{-\alpha_{n}}+\sum_{t=n-T}^{n} \mathbb{Q}\left(F_{n}, t=\tau_{\zeta_{n}\left(w_{n}\right)}, \tau_{\zeta_{n}}^{\left(2, w_{n}\right)}>n, \exists v \in \mathbb{B}\left(w_{t}\right), \min _{u \geq v,|u|=n} V(u) \leq \alpha_{n}-x\right)
\end{aligned}
$$

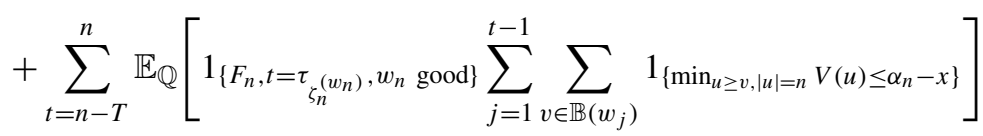

$$
\begin{aligned}
& =: \varepsilon \mathrm{e}^{-\alpha_{n}}+A_{(6.4)}+B_{(6.4)},
\end{aligned}
$$

with obvious definitions of $A_{(6.4)}$ and $B_{(6.4)}$, and where $A_{(6.4)}$ corresponds to the possibility $k=\tau_{\zeta_{n}}^{\left(w_{n}\right)}$ and $B_{(6.4)}$ to $k<\tau_{\zeta_{n}}^{\left(w_{n}\right)}$.

In what follows, we shall bound separately $A_{(6.4)}$ and $B_{(6.4)}$. We stress that the i.i.d. hypothesis on the point process, $\mathcal{L}=\sum_{i=1}^{v} \delta_{\left\{\xi_{i}\right\}}$ with $\left(\xi_{i}\right)$ i.i.d. and independent of $v$, will be only used in the estimation on $A_{(6.4)}$.

Firstly, we estimate $A_{(6.4)}$. Let $\Upsilon_{t}^{(n)}:=\left\{\left|V\left(w_{t-1}\right)-\mathrm{m}(n-1)\right| \leq \frac{n}{\log n},\left|V\left(w_{t}\right)-\left(\alpha_{n}-x\right)\right| \leq\right.$ $\log n\}$. For any $t \in[n-T, n]$, we remark that

$$
\begin{aligned}
& \mathbb{Q}\left(F_{n}, t=\tau_{\zeta_{n}^{\left(w_{n}\right)}}, \tau_{\zeta_{n}}^{\left(2, w_{n}\right)}>n,\left(\Upsilon_{t}^{(n)}\right)^{c}\right) \\
& \leq \mathbb{Q}\left(t=\tau_{\zeta_{n}\left(w_{n}\right)}, \tau_{\zeta_{n}}^{\left(2, w_{n}\right)}>n,\left|V\left(w_{t-1}\right)-\mathrm{m}(n-1)\right|>\frac{n}{\log n}\right) \\
& +\mathbb{Q}\left(V\left(w_{n}\right) \leq \alpha_{n}-x, \min _{\tau_{\zeta n}^{\left(w_{n}\right)} \leq i \leq n} V\left(w_{j}\right) \geq \alpha_{n}-x-L, t=\tau_{\zeta_{n}^{\left(w_{n}\right)}},\right. \\
& \left.\left|V\left(w_{t}\right)-\left(\alpha_{n}-x\right)\right|>\log n\right) .
\end{aligned}
$$


Recall that under $\mathbb{Q},\left(V\left(w_{j}\right)_{j \geq 0}\right)$ is distributed as the real-valued random walk $\left(S_{j}\right)_{j \geq 0}$ under P. Then an immediate application of (3.9) and (3.13) gives that for any $n$ large enough and $x \geq 0$,

$$
\sum_{t=n-T}^{n} \mathbb{Q}\left(F_{n}, t=\tau_{\zeta_{n}^{\left(w_{n}\right)}},\left(\Upsilon_{t}^{(n)}\right)^{c}\right) \leq \varepsilon \mathrm{e}^{-\alpha_{n}}
$$

Then we have

$$
\begin{aligned}
A_{(6.4)} \leq & \varepsilon \mathrm{e}^{-\alpha_{n}}+\sum_{t=n-T}^{n} \mathbb{Q}\left(\Upsilon_{t}^{(n)}, t=\tau_{\zeta_{n}^{\left(w_{n}\right)}}, \exists v \in \mathbb{B}\left(w_{t}\right), \min _{u \geq v,|u|=n} V(u) \leq \alpha_{n}-x\right) \\
\leq & \varepsilon \mathrm{e}^{-\alpha_{n}} \\
& +\sum_{t=0}^{T} z \sup _{z,|z+\operatorname{m} n| \leq 2 n / \log n} \mathbb{Q}\left(\left|V\left(w_{1}\right)-z\right| \leq \log n, \exists v \in \mathbb{B}\left(w_{1}\right), \min _{u \geq v,|u|=t} V(u) \leq-\frac{\mathrm{m}}{2} n\right) .
\end{aligned}
$$

For any $t \in \mathbb{N}$, let $\left(M_{t}^{(j)}\right)_{j \geq 0}$ a sequence of i.i.d. random variables distributed as $M_{t}$ the minimum of the branching random walk at time $t$. By Proposition 2.3, for any $z \in \mathbb{R}$, and the i.i.d. hypothesis we have

$$
\begin{aligned}
& \mathbb{Q}\left(\left|V\left(w_{1}\right)-z\right| \leq \log n, \exists v \in \mathbb{B}\left(w_{1}\right), \min _{u \geq v,|u|=t+1} V(u) \leq-\frac{\mathrm{m}}{2} n\right) \\
& =\mathbb{E}\left[\sum_{i=1}^{v} \mathrm{e}^{-\xi_{i}} 1_{\left\{\left|\xi_{i}-z\right| \leq \log n\right\}} 1_{\left\{\exists j \neq i, \xi_{j}+M_{t}^{(j)} \leq-(\mathrm{m} / 2) n\right\}}\right] \\
& =\sum_{k=1}^{\infty} \mathbb{P}(v=k) k \mathbb{E}\left[\mathrm{e}^{-\xi_{i}} 1_{\left\{\left|\xi_{i}-z\right| \leq \log n\right\}}\right] \mathbb{P}\left(\exists j \in\{1, \ldots, k-1\}, \xi_{j}+M_{t}^{(j)} \leq-\frac{\mathrm{m}}{2} n\right) .
\end{aligned}
$$

By (1.4), there exists a slowly varying function $\widetilde{\ell}(n)$ such that

$$
\sup _{z,|z+\mathrm{m} n| \leq 2 n / \log n} \mathbb{E}\left[\mathrm{e}^{-\xi_{i}} 1_{\left\{\left|\xi_{i}-z\right| \leq \log n\right\}}\right] \leq n^{-(\alpha+1)} \tilde{\ell}(n) .
$$

By Lemma 4.2, for any $k \geq 1$,

$$
\begin{aligned}
\mathbb{P}\left(\exists j \in\{1, \ldots, k-1\}, \xi_{j}+M_{t}^{(j)} \leq-\frac{\mathrm{m}}{2} n\right) & \leq k \mathbb{E}\left[\mathrm{e}^{-\xi_{1}-\mathrm{m} n / 2}\right] \\
& =\frac{k}{\mathbb{E}[v]} \mathrm{e}^{-(\mathrm{m} / 2) n}
\end{aligned}
$$

by using the fact that $\mathbb{E}\left[\mathrm{e}^{-\xi_{1}}\right]=\frac{1}{\mathbb{E}[v]}$. 
Combining these two inequalities then using (1.5) we get

$$
\begin{aligned}
& \mathbb{Q}\left(\left|V\left(w_{1}\right)-z\right| \leq \log n, \exists v \in \mathbb{B}\left(w_{1}\right), \min _{u \geq v,|u|=t} V(u) \leq-\frac{\mathrm{m}}{2} n\right) \\
& \quad \leq n^{-\alpha-1} \tilde{\ell}(n)\left(\sum_{k=1}^{\mathrm{e}^{(\mathrm{m} / 8) n}} k^{2} \mathrm{e}^{-(\mathrm{m} / 2) n}+\sum_{k=\mathrm{e}^{(\mathrm{m} / 8) n}}^{\infty} k \mathbb{P}(v=k)\right) \\
& \quad \leq n^{-\alpha-1} \tilde{\ell}(n)\left(\mathrm{e}^{-(\mathrm{m} / 4) n}+\mathbb{E}\left(v 1_{\left.\left\{v \geq \mathrm{e}^{(\mathrm{m} / 8) n}\right)\right)}\right.\right. \\
& \quad \leq c n^{-\alpha-1} \tilde{\ell}(n)\left(\mathrm{e}^{-(\mathrm{m} / 4) n}+n^{-1}\right) .
\end{aligned}
$$

In addition to (6.5), it yields for any $\varepsilon>0$,

$$
A_{(6.4)} \leq 2 \varepsilon \mathrm{e}^{-\alpha_{n}}
$$

once $n$ is large enough.

Now we shall treat $B_{(6.4)}$. By the spinal decomposition (Proposition 2.3(iii)), for any $t \in$ $[n-T, n], j \in[1, t)$ and $v \in \mathbb{B}\left(w_{j}\right)$, conditionally on $\mathcal{G}=\sigma\left\{u, \Delta V(u), \overleftarrow{u}=w_{j}, j \geq 0\right\}$ and on $\{V(v)=b\}$, we have

$$
\mathbb{Q}\left(\min _{u \geq v,|u|=n} V(u) \leq \alpha_{n}-x \mid \mathcal{G}\right)=\mathbb{P}\left(M_{n-j} \leq \alpha_{n}-x-b\right) .
$$

If $j \leq \frac{2 n}{3}$, we apply Proposition 4.1 to get that $\mathbb{P}\left(M_{n-j} \leq \alpha_{n}-x-b\right) \leq K \mathrm{e}^{-\left(b+x+\alpha_{n-j}-\alpha_{n}\right)}$, whereas if $\frac{2 n}{3}<j \leq t$, we apply Lemma 4.2 (which holds obviously for all $x \in \mathbb{R}$ ) and get that $\mathbb{P}\left(M_{n-j} \leq \alpha_{n}-x-b\right) \leq \mathrm{e}^{-\left(b+x-\alpha_{n}\right)}$. Taking into account the fact that $w_{n}$ is good, we obtain

$$
\mathbf{E}_{\mathbb{Q}}\left[\sum_{v \in \mathbb{B}\left(w_{j}\right)} 1_{\left\{\min _{u \geq v,|u|=n} V(u) \leq \alpha_{n}-x\right\}} \mid \mathcal{G}\right] \leq \begin{cases}2 K \mathrm{e}^{\alpha_{n}-\alpha_{n-j}} \mathrm{e}^{B-x}, & \text { if } j \leq J, \\ K \mathrm{e}^{\alpha_{n}-\alpha_{n-j}} \mathrm{e}^{-j}{ }^{\varrho}, & \text { if } j \in\left(J, \frac{2}{3} n\right], \\ \mathrm{e}^{\alpha_{n}} \mathrm{e}^{-j^{\varrho}}, & \text { if } j \in\left(\frac{2}{3} n, t\right) .\end{cases}
$$

By summing these inequalities, for $n$ large enough we get that

$$
\begin{aligned}
B_{(6.4)} & \leq K^{\prime}\left(J \mathrm{e}^{B-x}+\mathrm{e}^{-J^{\varrho / 2}}\right) \sum_{t=n-T}^{n} \mathbb{Q}\left(F_{n}, t=\tau_{\zeta_{n}}\left(w_{n}\right), w_{n} \text { good }\right) \\
& \leq K^{\prime}\left(J \mathrm{e}^{B-x}+\mathrm{e}^{-J^{\varrho / 2}}\right) \sum_{t=n-T}^{n} \mathbf{P}\left(S_{n} \leq \alpha_{n}-x, \min _{\tau_{\zeta n} \leq i \leq n} S_{i} \geq \alpha_{n}-x-L, t=\tau_{\zeta_{n}}, \tau_{\zeta_{n}}^{(2)}>n\right) \\
& \leq K^{\prime \prime}\left(J \mathrm{e}^{B-x}+\mathrm{e}^{-J^{\varrho / 2}}\right)(1+L) T \mathrm{e}^{-\alpha_{n}},
\end{aligned}
$$

where for the second inequality we have used (4.2) for $F_{n} \cap\left\{t=\tau_{\zeta_{n}}\left(w_{n}\right)\right\}$ and for the last inequality, we have applied (3.15) to $y=\alpha_{n}-x-L$ and $a=1, \ldots,\lceil L\rceil$. Finally, we choose 
$J=J\left(L, T, K^{\prime \prime}\right)$ large enough and $x \geq x_{1}(B, J)$ so that $K^{\prime \prime}\left(J \mathrm{e}^{B-x}+\mathrm{e}^{-J^{\varrho / 2}}\right)(1+L) T \leq \varepsilon$. Then $B_{(6.4)} \leq \varepsilon \mathrm{e}^{-\alpha_{n}}$ and (5.3) follows. This proves Proposition 5.1.

It remains us to prove Lemma 6.1. For any integers $k \leq n$, define $\underline{S}_{[k, n]}:=\min _{j \in[k, n]} S_{j}$.

Proof of Lemma 6.1. Firstly, we will prove that with overwhelming probability the trajectory of $\left(V\left(w_{i}\right)\right)_{i \geq 0}$ contains only one big jump and never drops too low. Recall the notation $F_{n}$ defined in (6.3). Write for brevity

$$
y:=\alpha_{n}-x-L .
$$

We shall use several times the fact that under $\mathbb{Q},\left(V\left(w_{j}\right), j \geq 0\right)$ has the same law as $\left(S_{j}, j \geq 0\right)$ under P. Then by (3.14) with $a=0,1, \ldots,\lceil L\rceil$, we get that for some constant $K_{L}>0$ depending on $L$,

$$
\begin{aligned}
\mathbb{Q}\left(F_{n}, \tau_{\zeta_{n}}^{\left(2, w_{n}\right)} \leq n\right) & =\sum_{i=n-T}^{n} \mathbf{P}\left(\underline{S}_{\left[\tau_{\zeta n}, n\right]} \geq y, \tau_{\zeta_{n}}=i, S_{n} \in[y, y+L], \tau_{\zeta_{n}}^{(2)} \leq n\right) \\
& \leq K_{L} \sum_{i=n-T}^{n} n^{-2 \alpha} i^{-1 / \alpha} \ell_{3}(n) \leq \varepsilon \mathrm{e}^{-\alpha_{n}},
\end{aligned}
$$

for all large $n$. We claim that there exists some positive constant $c_{4}=c_{4}(L, T)$ such that for all $n$ large,

$$
\mathbb{Q}\left(F_{n}, \min _{1 \leq j<\tau_{\zeta n}^{\left(w_{n}\right)}} V\left(w_{j}\right) \leq-c_{4}, \tau_{\zeta_{n}}^{\left(2, w_{n}\right)}>n\right) \leq O(\varepsilon) \mathrm{e}^{-\alpha_{n}} .
$$

Let us denote by $\mathbb{Q}_{(6.7)}$ the probability term in (6.7). Denote by $j$ be the first time such that $V\left(w_{j}\right) \leq-c_{4}$; then by using the Markov property at $j$, we get that

$$
\begin{aligned}
\mathbb{Q}(6.7)= & \sum_{i=n-T}^{n} \sum_{j=1}^{i-1} \mathbf{E}\left[1_{\left\{\underline{S}_{j-1}>-c_{4}, S_{j} \leq-c_{4}, \min _{k \leq j} X_{k} \geq-\zeta_{n}\right\}}\right. \\
& \left.\times \mathbf{P}_{S_{j}}\left(S_{n-j} \leq y+L, \min _{\tau_{\zeta n} \leq i \leq n-j} S_{i} \geq y, \tau_{\zeta_{n}}=i-j, \tau_{\zeta_{n}}^{(2)}>n-j\right)\right] .
\end{aligned}
$$

If $j \geq \frac{n}{2}$ by Lemma 3.1 (with $x=\mathrm{m} j+c_{4}$ and $y=\zeta_{n}$ there) we get that

$$
\mathbf{P}\left(S_{j} \leq-c_{4}, \min _{k \leq j} X_{k} \geq-\zeta_{n}\right) \leq K \mathrm{e}^{-(\log n)^{3} / K},
$$

whereas for $j \leq \frac{n}{2}$, since $y-S_{j} \leq y+c_{4}+\zeta_{n} \leq \frac{\mathrm{m}}{2}(n-j)$, by using $\lceil L\rceil$ times (3.15) (with $a \in[0, L]$ being integer), we deduce that for any $i \in[n-T, n]$ and on $\left\{S_{j} \leq-c_{4}, \min _{k \leq j} X_{k} \geq\right.$ $\left.-\zeta_{n}\right\}$

$$
\mathbf{P}_{S_{j}}\left(S_{n-j} \leq y+L, \underline{S}_{\left[\tau_{\zeta_{n}}, n-j\right]} \geq y, \tau_{\zeta_{n}}=i-j, \tau_{\zeta_{n}}^{(2)}>n-j\right) \leq K^{\prime}(1+L) \mathrm{e}^{-\alpha_{n}}
$$


(we used the fact that $\mathrm{e}^{-\alpha_{n-j}}=O\left(\mathrm{e}^{-\alpha_{n}}\right)$ when $\left.j \leq \frac{n}{2}\right)$. It follows that

$$
\begin{aligned}
\mathbb{Q}(6.7) & \leq \sum_{i=n-T}^{n} \sum_{j=n / 2}^{i-1} K \mathrm{e}^{-(\log n)^{3} / K}+K^{\prime}(1+L) \mathrm{e}^{-\alpha_{n}} \sum_{i=n-T}^{n} \sum_{j=1}^{n / 2} \mathbf{P}\left(\underline{S}_{j-1} \geq-c_{4}, S_{j}<-c_{4}\right) \\
& \leq \varepsilon \mathrm{e}^{-\alpha_{n}}+K^{\prime}(1+L) T \mathrm{e}^{-\alpha_{n}} \sum_{j=1}^{n / 2} \mathbf{P}\left(\underline{S}_{j-1} \geq-c_{4}, S_{j}<-c_{4}\right) \\
& \leq \varepsilon \mathrm{e}^{-\alpha_{n}}+K^{\prime}(1+L) T \mathrm{e}^{-\alpha_{n}} \mathbf{P}\left(\min _{k \geq 0} S_{k}<-c_{4}\right) \\
& \leq 2 \varepsilon \mathrm{e}^{-\alpha_{n}}
\end{aligned}
$$

by choosing $c_{4}=c_{4}(L, T)$ large enough to get the last inequality. Then (6.7) follows.

By combining (6.6) and (6.7), to get Lemma 6.1 it is enough to prove the following assertion: for any $L, T, \varepsilon>0$ there exist $B>0$ and $J$ such that for any $n \geq n_{0}(J, B, L, T, \varepsilon), x \geq 0$,

$$
\mathbb{Q}\left(F_{n}, \min _{1 \leq j<\tau_{\zeta_{n}}^{\left(w_{n}\right)}} V\left(w_{j}\right) \geq-c_{4}, \tau_{\zeta_{n}}^{\left(2, w_{n}\right)}>n, w_{n} \text { not good }\right) \leq O(\varepsilon) \mathrm{e}^{-\alpha_{n}}
$$

Recall that $0<\varrho<\min \left(\frac{\alpha-1}{2}, \frac{1}{12}\right)$. Before establishing (6.9), we prove the following claim.

Claim 6.2. (i) There is a sequence of positive real numbers $\left(\varepsilon_{j}\right)$ such that $\lim _{j \rightarrow \infty} \varepsilon_{j}=0$ and for any integer $j$ and $z \in \mathbb{R}, y \geq 0$,

$$
\sum_{p=j}^{\infty} \mathbf{P}\left(S_{p}-p^{\varrho} \leq z, \underline{S}_{p} \geq-y\right) \leq \frac{10}{\mathrm{~m}}\left(z-\frac{\mathrm{m} j}{10}\right)^{+}+\varepsilon_{j}\left(1+y+z^{+}\right) .
$$

(ii) There exists some positive constant $K_{L, T}>0$ such that for all large $n$ and $k \in[1, n-T)$,

$$
\begin{aligned}
& \sup _{z \leq-\mathrm{m} n / 5} \mathbf{P}\left(S_{n-k} \leq z, \min _{\tau_{\zeta n} \leq i \leq n-k} S_{i} \geq z-L, \tau_{\zeta_{n}} \in[n-k-T, n-k], \tau_{\zeta_{n}}^{(2)}>n-k\right) \\
& \quad \leq K_{L, T} \mathrm{e}^{-\alpha_{n}} .
\end{aligned}
$$

Proof. (i) Observe that

$$
\begin{aligned}
& \sum_{p \geq j} \mathbf{P}\left(S_{p}-p^{\varrho} \leq z, \underline{S}_{p} \geq-y\right) \\
& \quad \leq\left(\frac{10}{\mathrm{~m}} z-j\right)^{+}+\sum_{p \geq \max (10 z / \mathrm{m}, j)} \mathbf{P}\left(S_{p} \in\left[-y, z+p^{\varrho}\right]\right)
\end{aligned}
$$


Then observe that $z+p^{\varrho} \leq \frac{\mathrm{m}}{2} p$ for all $p \geq \frac{10 z}{\mathrm{~m}}$ and $p \geq j_{0}$ if $j_{0}$ is large enough. By applying Lemma 3.4, we get

$$
\begin{aligned}
\sum_{p \geq j} \mathbf{P}\left(S_{p}-p^{\varrho} \leq z, \underline{S}_{p} \geq-y\right) & \leq \frac{10}{\mathrm{~m}}\left(z-\frac{\mathrm{m} j}{10}\right)^{+}+K \sum_{p \geq \max (10 z / \mathrm{m}, j)} \frac{l(p)\left(y+z^{+}+p^{\varrho}\right)}{p^{\alpha}} \\
& \leq \frac{10}{\mathrm{~m}}\left(z-\frac{\mathrm{m} j}{10}\right)^{+}+\varepsilon_{j}\left(1+y+z^{+}\right),
\end{aligned}
$$

with $\varepsilon=O\left(j^{(1-\alpha) / 2}\right)$, proving (6.10).

(ii) Denote by $\mathbf{P}_{(6.11)}$ the probability term in (6.11). Then

$$
\begin{aligned}
\mathbf{P}_{(6.11)} & =\sum_{j=n-k-T}^{n-k} \mathbf{P}\left(S_{n-k} \leq z, \min _{j \leq i \leq n-k} S_{i} \geq z-L, \tau_{\zeta_{n}}=j, \tau_{\zeta_{n}}^{(2)}>n-k\right) \\
= & \sum_{j=n-k-T}^{n-k} \mathbf{P}_{(6.11)}(j) .
\end{aligned}
$$

Notice that $z-L \leq S_{n-k} \leq z$. Therefore, if $S^{\prime}:=S_{n-k}-X_{j} \geq-\frac{\mathrm{m}}{10} n$ then $X_{j} \leq z+\frac{\mathrm{m}}{10} n \leq$ $-\frac{\mathrm{m}}{10} n$. Moreover, $z-L-S^{\prime} \leq X_{j} \leq z-S^{\prime}$. By the independence of $X_{j}$ and $S^{\prime}$, we get that

$$
\mathbf{P}\left(z-L \leq S_{n-k} \leq z, \tau_{\zeta_{n}}=j, S^{\prime} \geq-\frac{\mathrm{m}}{10} n\right) \leq \sup _{b \leq-(\mathrm{m} / 10) n} \mathbf{P}\left(X_{j} \in[b-L, b]\right) \leq K_{L} \mathrm{e}^{-\alpha_{n}},
$$

by using the density of $X_{j}$ given by (1.4). On the other hand, if $S^{\prime}:=S_{n-k}-X_{j}<-\frac{\mathrm{m}}{10} n$ then we can apply Lemma 3.1 to see that

$\mathbf{P}\left(S^{\prime}<-\frac{\mathrm{m}}{10} n, \tau_{\zeta_{n}}=j, \tau_{\zeta_{n}}^{(2)}>n-k\right) \leq \mathbf{P}\left(S_{n-k-1}<-\frac{\mathrm{m}}{10} n, \tau_{\zeta_{n}}>n-k-1\right) \leq K \mathrm{e}^{-\mathrm{m} n /\left(10 \zeta_{n}\right)}$.

Therefore, $\mathbf{P}_{(6.11)}(j) \leq K_{L} \mathrm{e}^{-\alpha_{n}}+K \mathrm{e}^{-m n /\left(10 \zeta_{n}\right)}$ and (6.11) follows if we take $K_{L, T}=2(1+$ T) $K_{L}$. This completes the proof of Claim 6.2.

Let us go back to the proof of (6.9). Define for any $k \geq 1, \xi\left(w_{k}\right):=\sum_{v \in \mathbb{B}\left(w_{k}\right)} \mathrm{e}^{-\Delta V(v)}$. Then

$$
\sum_{v \in \mathbb{B}\left(w_{k}\right)} \mathrm{e}^{-(V(v)+x)}=\mathrm{e}^{-V\left(w_{k-1}\right)-x} \xi\left(w_{k}\right)
$$

Notice that the sequence $\left\{\xi\left(w_{k}\right), \Delta V\left(w_{k}\right)\right\}_{k \geq 1}$ are i.i.d. under $\mathbb{Q}$. Define $\xi=\xi\left(w_{1}\right)$.

Let $n$ be large enough so that $n-T>J$. On $\left\{\tau_{\zeta_{n}}^{\left(2, w_{n}\right)}>n\right\} \cap\left\{w_{n}\right.$ not good $\}$, either there is some $1 \leq k \leq J$ such that $\xi\left(w_{k}\right)>\mathrm{e}^{B+V\left(w_{k-1}\right)}$ or some $J<k<\tau_{\zeta_{n}}^{\left(w_{n}\right)}$ such that $\xi\left(w_{k}\right)>$ $\mathrm{e}^{V\left(w_{k-1}\right)+x-k^{\varrho}}$. We discuss separately these two cases and give the precise choices of $J$ and $B$ :

The first case: $J<k<\tau_{\zeta_{n}}^{\left(w_{n}\right)}$ and the choice of $J$. 
Notice that $\tau_{\zeta_{n}}^{\left(w_{n}\right)} \in[n-T, n]$. For any $J<k<n-T$, we apply the Markov property at $k$ to arrive at

$$
\begin{aligned}
& \mathbb{Q}_{(6.13)}(k) \\
& \quad:=\mathbb{Q}\left(k<\tau_{\zeta_{n}}^{\left(w_{n}\right)}, \xi\left(w_{k}\right)>\mathrm{e}^{x+V\left(w_{k-1}\right)-k^{\varrho}}, \min _{1 \leq j \leq k} V\left(w_{j}\right) \geq-c_{4}, F_{n}, \tau_{\zeta_{n}}^{\left(2, w_{n}\right)}>n\right) \\
& \quad=\mathbb{E}_{\mathbb{Q}}\left[1_{\left\{\xi\left(w_{k}\right)>\mathrm{e}^{x+V\left(w_{k-1}\right)-k}{ }^{\varrho}, \min _{1 \leq j \leq k} V\left(w_{j}\right) \geq-c_{4}, \min _{j \leq k} \Delta V\left(w_{j}\right) \geq-\zeta_{n}\right\}} g_{n-k}\left(V\left(w_{k}\right)\right)\right],
\end{aligned}
$$

with

$$
\begin{aligned}
g_{n-k}(b):= & \mathbf{P}\left(S_{n-k} \leq y-b+L, \min _{\tau_{\zeta_{n}} \leq j \leq n-k} S_{j} \geq y-b,\right. \\
& \left.\tau_{\zeta_{n}} \in[n-k-T, n-k], \tau_{\zeta_{n}}^{(2)}>n-k\right), \quad z \in \mathbb{R} .
\end{aligned}
$$

When $k \leq \frac{n}{2}$, we can apply (3.12) to get that for $b:=V\left(w_{k}\right) \geq-c_{4}$,

$$
g_{n-k}(b) \leq K_{L}^{\prime} \mathrm{e}^{-\alpha_{n}},
$$

with some positive constant $K_{L}^{\prime}$ depending on $L$ [in fact $K_{L}^{\prime}=O\left(L^{2}\right)$ ].

For $k \geq \frac{n}{2}$, if $b:=V\left(w_{k}\right)>\frac{\mathrm{m} n}{4}$, then $y+L-b \leq \frac{-\mathrm{m} n}{5}$ and $g_{n-k}(b) \leq K_{L, T} \mathrm{e}^{-\alpha_{n}}$ by (6.11). Consequently, we get that for any $J<k<n-T$ and $x \geq 0$,

$$
\begin{aligned}
& \mathbb{Q}_{(6.13)}(k) \leq K_{L, T}^{\prime \prime} \mathrm{e}^{-\alpha_{n}} \mathbb{Q}\left(\xi\left(w_{k}\right)>\mathrm{e}^{V\left(w_{k-1}\right)-k^{\varrho}}\right) \\
& +1_{\{k \geq n / 2\}} \mathbb{Q}\left(V\left(w_{k}\right) \leq \frac{\mathrm{m} n}{4}, \min _{j \leq k} \Delta V\left(w_{j}\right) \geq-\zeta_{n}\right) .
\end{aligned}
$$

Moreover, $\mathbb{Q}\left(V\left(w_{k}\right) \leq \frac{\mathrm{m} n}{4}, \min _{j \leq k} \Delta V\left(w_{j}\right) \geq-\zeta_{n}\right)=\mathbf{P}\left(S_{k} \leq \frac{\mathrm{m} n}{4}, \min _{j \leq k} X_{j} \geq-\zeta_{n}\right) \leq$ $K \mathrm{e}^{-\mathrm{mn} /\left(4 \zeta_{n}\right)}$ by Lemma 3.1. Since under $\mathbb{Q}, \xi\left(w_{k}\right)$ is independent of $V\left(w_{k-1}\right)$ which is distributed as $S_{k-1}$ under $\mathbf{P}$, and, moreover, $\xi\left(w_{k}\right)$ has the same law as $\xi$, it follows from (6.10) that

$$
\begin{aligned}
\sum_{J<k \leq n-T} \mathbb{Q}(6.13)(k) \leq & K_{T, L}^{\prime \prime} \mathrm{e}^{-\alpha_{n}} \mathbb{E}_{\mathbb{Q}}\left[\sum_{J<k \leq n-T} \mathbf{P}\left(\log \xi\left(w_{k}\right) \geq S_{k-1}-k^{\varrho}, \underline{S}_{k-1} \geq-c_{4}\right)\right] \\
& +K n \mathrm{e}^{-\mathrm{m} n /\left(4 \zeta_{n}\right)} \\
\leq & K_{T, L} \mathrm{e}^{-\alpha_{n}}\left(\mathbb{E}_{\mathbb{Q}}\left[\left(\log \xi-\frac{\mathrm{m} J}{10}\right)^{+}\right]+\varepsilon_{J} \mathbb{E}_{\mathbb{Q}}\left[1+c_{4}+(\log \xi)^{+}\right]\right) \\
& +K n \mathrm{e}^{-\mathrm{m} n /\left(4 \zeta_{n}\right)}
\end{aligned}
$$

with $\varepsilon_{J} \rightarrow 0$ as $J \rightarrow \infty$. By (1.5), $\mathbb{E}_{\mathbb{Q}}\left[(\log \xi)^{+}\right] \leq \mathbb{E}\left[\sum_{|u|=1} \mathrm{e}^{-V(u)}\left(\log \left[\sum_{|u|=1} \mathrm{e}^{-V(u)}\right]\right)^{+}\right]<$ $\infty$, thus we choose and then fix $J=J(\varepsilon, T, L)$ large enough so that $\mathbb{E}_{\mathbb{Q}}\left[\left(\log \xi-\frac{m J}{10}\right)^{+}\right]+$ 
$\varepsilon_{J} \mathbb{E}_{\mathbb{Q}}\left[1+c_{4}+(\log \xi)^{+}\right] \leq \frac{\varepsilon}{K_{L, T}}$. Then for all large $n$, we get that

$$
\sum_{J<k \leq n-T} \mathbb{Q}_{(6.13)}(k) \leq 2 \varepsilon \mathrm{e}^{-\alpha_{n}} .
$$

The second (and last) case: $1 \leq k \leq J$ and the choice of $B$.

Under $\mathbb{Q}$ and conditionally on $\left\{V\left(w_{k}\right)=z\right\}$, the process $\left\{V\left(w_{i+k}\right), 0 \leq i \leq n-k\right\}$ is distributed as $\left\{S_{i}, 0 \leq i \leq n-k\right\}$ under $\mathbf{P}_{z}$. It follows from the Markov property at $k$ that

$$
\begin{aligned}
\mathbb{Q} & \left(\xi\left(w_{k}\right) \geq \mathrm{e}^{B+V\left(w_{k-1}\right)}, F_{n}, \min _{1 \leq j \leq k} V\left(w_{j}\right) \geq-c_{4}, \tau_{\zeta_{n}}^{\left(2, w_{n}\right)}>n\right) \\
= & \mathbb{E}_{\mathbb{Q}}\left[1_{\left\{\xi\left(w_{k}\right) \geq \mathrm{e}^{B+V\left(w_{k-1}\right)}, \min _{1 \leq j \leq k} V\left(w_{j}\right) \geq-c_{4}\right\}}\right. \\
\quad & \left.\times \mathbf{P}_{V\left(w_{k}\right)}\left(S_{n-k} \leq y+L, \min _{\tau_{\zeta n}-k \leq j \leq n-k} S_{j} \geq y, \tau_{\zeta_{n}} \in[n-k-T, n-k], \tau_{\zeta_{n}}^{(2)}>n-k\right)\right] \\
\leq & K_{L} \mathbb{Q}\left(\xi\left(w_{k}\right) \geq \mathrm{e}^{B+V\left(w_{k-1}\right)}, \min _{1 \leq j \leq k} V\left(w_{j}\right) \geq-c_{4}\right) \mathrm{e}^{-\alpha_{n}},
\end{aligned}
$$

where $K_{L}>0$ denotes some constant depending on $L$ and we have applied (3.12) to get the last inequality [remark that $\left.y-V\left(w_{k}\right) \leq y+c_{4} \leq \frac{m}{2}(n-k)\right]$. Furthermore,

$$
\begin{aligned}
& \mathbb{E}_{\mathbb{Q}}\left[\sum_{k=1}^{J} 1_{\left\{\log \xi\left(w_{k}\right) \geq B+V\left(w_{k-1}\right), \min _{1 \leq j \leq k} V\left(w_{j}\right) \geq-c_{4}\right\}}\right] \\
& \leq \sum_{k=1}^{J} \mathbb{Q}\left(V\left(w_{k-1}\right) \leq-\frac{B}{2}\right)+\sum_{k=1}^{J} \frac{2}{B} \mathbb{E}_{\mathbb{Q}}\left[\left(\log \left(\xi\left(w_{k}\right)\right)\right)^{+}\right] \\
& =\sum_{k=1}^{J} \mathbf{P}\left(S_{k-1} \leq-\frac{B}{2}\right)+\frac{2 J}{B} \mathbb{E}_{\mathbb{Q}}\left[(\log \xi)^{+}\right] \\
& \leq \frac{\varepsilon}{K_{L}}
\end{aligned}
$$

by choosing $B=B(J, L, T, \varepsilon)$ large enough. Finally, we have

$$
\mathbb{Q}\left(\exists k \in[1, J]: \xi\left(w_{k}\right) \geq \mathrm{e}^{B+V\left(w_{k-1}\right)}, \min _{1 \leq j \leq k} V\left(w_{j}\right) \geq-c_{4}, F_{n}, \tau_{\zeta_{n}}^{\left(2, w_{n}\right)}>n\right) \leq \varepsilon \mathrm{e}^{-\alpha_{n}}
$$

By combining (6.14) and (6.15), we get (6.9) and, therefore, complete the proof of Lemma 6.1. 


\section{Appendix}

The convergence in law in Theorem 1.1 holds in fact under a setting more general than the i.i.d. case. As mentioned before, the main contribution to the renormalization $M_{n}-\alpha_{n}$ comes from the large drop which is unique in the i.i.d. case. However, in the general case, nothing prevents from the possible simultaneous large drops (in the same generation), this is why we need some assumptions.

The main additional hypothesis, which ensures the convergence of the relative displacements at the large drop time, is stated as follows: Assume that

$$
\begin{aligned}
& \text { For any } f: \mathbb{R} \rightarrow \mathbb{R}_{+} \text {measurable with compact support } \\
& \qquad \lim _{z \rightarrow-\infty} \mathbb{E}_{\mathbb{Q}}\left[\mathrm{e}^{-\sum_{v \in \mathbb{B}\left(w_{1}\right)} f\left(V\left(w_{1}\right)-V(v)\right)} \mid V\left(w_{1}\right)=z\right] \rightarrow \int \Xi(\mathrm{d} \theta) \mathrm{e}^{-\langle f, \theta\rangle},
\end{aligned}
$$

where $\mathbb{B}\left(w_{1}\right)$ is defined in (1.9), $\Xi$ is the distribution of some point process on $\mathbb{R} \cup\{-\infty\}$ and we use the notation $\langle f, \theta\rangle:=\int_{\mathbb{R}} f(x) \theta(\mathrm{d} x)$ for any $\theta \in \mathcal{M}$, the space of $\sigma$-finite measures on $\mathbb{R} \cup\{-\infty\}$. For instance, when $\mathscr{L}=\sum_{i=1}^{v} \delta_{\left\{\xi_{i}\right\}}$ with $\left(\xi_{i}\right)$ i.i.d. and independent of $v$ it is easily seen that (A.1) holds with $\Xi=\delta_{\{-\infty\}}$.

In view of the compact support hypothesis of $f$ in (A.1), we also need two other technical hypotheses in the truncation argument:

$$
\begin{aligned}
& \text { Under } \mathbb{Q} \text {, as } z \rightarrow-\infty \text {, the laws of } \# \mathbb{B}\left(w_{1}\right) \text { conditionally on }\left\{V\left(w_{1}\right)=z\right\} \text { are tight, } \\
& \lim _{\lambda \rightarrow \infty} \limsup _{z \rightarrow-\infty} \mathbb{Q}\left(\bigcup_{v \in \mathbb{B}\left(w_{1}\right)}\left\{V\left(w_{1}\right)-V(v) \geq \lambda\right\} \mid V\left(w_{1}\right)=z\right) \rightarrow 0
\end{aligned}
$$

The assumptions (A.1), (A.2) and (A.3) hold for a variety of situations, for instance, in addition to the i.i.d. case and the case in Remark 1.4, here is another simple example: $\mathscr{L}=\sum_{i=1}^{3} \delta_{\left\{\xi_{i}\right\}}$ with $\xi_{1}$ an independent copy of $\xi_{2}$ and $\xi_{3}=\xi_{2}$ a.s., it is easy to check that (A.1), (A.2) and (A.3) are satisfied with $\Xi=\frac{1}{3} \delta_{\{-\infty\}}+\frac{2}{3} \delta_{\{0\}}$. However, we do not know how to describe these assumptions in a completely general setting under the original probability $\mathbb{P}$.

We have the following convergence in law of $M_{n}-\alpha_{n}$ in the general case:

Theorem A.3. Assume (1.1), (1.4) and (1.5), as well as (A.1), (A.2) and (A.3). Then for any $x \in \mathbb{R}$,

$$
\lim _{n \rightarrow \infty} \mathbb{P}\left(M_{n} \geq \alpha_{n}+x\right)=\mathbb{E}\left(\exp \left(-c \mathrm{e}^{x} W_{\infty}\right)\right)
$$

where $c>0$ is some finite constant.

As in the i.i.d. case, the key estimate in the proof of Theorem A.3 is to establish Proposition 2.1, with some positive constant $c$ instead of $c_{*}$, under the assumptions (A.1), (A.2) and (A.3). To this end, as mentioned in Remark 5.2, we firstly modify the definition of $\mathcal{E}_{n}(x)$ in (5.2) by replacing $k \leq \tau_{\zeta_{n}}^{\left(w_{n}\right)}$ by $k<\tau_{\zeta_{n}}^{\left(w_{n}\right)}$ so that (5.3) still holds. The main difference comes from 
the expression of $A_{(5.5)}(k)$ : Because of the possibility of large drops from the particles in $\mathbb{B}\left(w_{k}\right)$ [here $k=\tau_{\zeta_{n}}^{\left(w_{n}\right)}$ is the large drop time], $A_{(5.5)}(k)$ should be replaced by

$$
\mathrm{e}^{V\left(w_{n}\right)} \frac{1_{\left\{M_{n}=V\left(w_{n}\right) \leq \alpha_{n}-x, \min _{k \leq j \leq n} V\left(w_{j}\right) \geq \alpha_{n}-x-L, \tau_{\zeta n}^{\left(w_{n}\right)}=k\right\}}}{\sum_{\overleftarrow{v}=w_{k-1}} \sum_{|u|=n, u \in \mathbb{T}^{(v)}} 1_{\left\{V(u)=V\left(w_{n}\right)\right\}}}
$$

By using (A.1), we are able to estimate the contribution of these $v \in \mathbb{B}\left(w_{k}\right)$ to $\sum_{|u|=n, u \in \mathbb{T}^{(v)}} \times$ $1_{\left\{V(u)=V\left(w_{n}\right)\right\}}$ and to $\left\{M_{n}=V\left(w_{n}\right)\right\}$, after a truncation argument based on (A.2) and (A.3). Finally, the constant $c$ will follow from an application of the renewal result Lemma 3.6. We feel free to omit the details.

\section{Acknowledgements}

We are grateful to an anonymous referee for the careful reading on the manuscript and helpful suggestions on the presentation of this paper. Research partially supported by ANR (MEMEMOII) 2010 BLAN 0125.

\section{References}

[1] Addario-Berry, L. and Reed, B. (2009). Minima in branching random walks. Ann. Probab. 371044 1079. MR2537549

[2] Aïdékon, E. (2013). Convergence in law of the minimum of a branching random walk. Ann. Probab. 41 1362-1426. MR3098680

[3] Alsmeyer, G., Biggins, J.D. and Meiners, M. (2012). The functional equation of the smoothing transform. Ann. Probab. 40 2069-2105. MR3025711

[4] Attia, N. and Barral, J. (2014). Hausdorff and packing spectra, large deviations, and free energy for branching random walks in $\mathbb{R}^{d}$. Comm. Math. Phys. 331 139-187. MR3231998

[5] Bacry, E. and Muzy, J.F. (2003). Log-infinitely divisible multifractal processes. Comm. Math. Phys. 236 449-475. MR2021198

[6] Barral, J., Kupiainen, A., Nikula, M., Saksman, E. and Webb, C. (2014). Critical Mandelbrot cascades. Comm. Math. Phys. 325 685-711. MR3148099

[7] Barral, J. and Mandelbrot, B.B. (2002). Multifractal products of cylindrical pulses. Probab. Theory Related Fields 124 409-430. MR1939653

[8] Barral, J., Rhodes, R. and Vargas, V. (2012). Limiting laws of supercritical branching random walks. C. R. Math. Acad. Sci. Paris 350 535-538. MR2929063

[9] Biggins, J.D. (1976). The first- and last-birth problems for a multitype age-dependent branching process. Adv. in Appl. Probab. 8 446-459. MR0420890

[10] Biggins, J.D. (1977). Martingale convergence in the branching random walk. J. Appl. Probab. 14 25-37. MR0433619

[11] Biggins, J.D. (1992). Uniform convergence of martingales in the branching random walk. Ann. Probab. 20 137-151. MR1143415

[12] Biggins, J.D. and Kyprianou, A.E. (1997). Seneta-Heyde norming in the branching random walk. Ann. Probab. 25 337-360. MR1428512 
[13] Biggins, J.D. and Kyprianou, A.E. (2004). Measure change in multitype branching. Adv. in Appl. Probab. 36 544-581. MR2058149

[14] Biggins, J.D. and Kyprianou, A.E. (2005). Fixed points of the smoothing transform: The boundary case. Electron. J. Probab. 10 609-631. MR2147319

[15] Bramson, M., Ding, J. and Zeitouni, O. (2013). Convergence in law of the maximum of the twodimensional discrete Gaussian free field. Preprint. Available at http://arxiv.org/abs/1301.6669.

[16] Bramson, M., Ding, J. and Zeitouni, O. (2014). Convergence in law of the maximum of nonlattice branching random walk. Preprint. Available at http://arxiv.org/abs/1404.3423.

[17] Bramson, M. and Zeitouni, O. (2009). Tightness for a family of recursion equations. Ann. Probab. 37 615-653. MR2510018

[18] Chauvin, B., Rouault, A. and Wakolbinger, A. (1991). Growing conditioned trees. Stochastic Process. Appl. 39 117-130. MR1135089

[19] Chen, X. (2015). A necessary and sufficient condition for the nontrivial limit of the derivative martingale in a branching random walk. Adv. in Appl. Probab. 47 741-760. MR3406606

[20] Collet, P. and Koukiou, F. (1992). Large deviations for multiplicative chaos. Comm. Math. Phys. 147 329-342. MR1174416

[21] Denisov, D., Dieker, A.B. and Shneer, V. (2008). Large deviations for random walks under subexponentiality: The big-jump domain. Ann. Probab. 36 1946-1991. MR2440928

[22] Derrida, B. and Spohn, H. (1988). Polymers on disordered trees, spin glasses, and traveling waves. J. Stat. Phys. 51 817-840. MR0971033

[23] Ding, J., Roy, R. and Zeitouni, O. (2015). Convergence of the centered maximum of log-correlated Gaussian fields. Preprint. Available at http://arxiv.org/abs/1503.04588.

[24] Durrett, R. and Liggett, T.M. (1983). Fixed points of the smoothing transformation. Z. Wahrsch. Verw. Gebiete 64 275-301. MR0716487

[25] Fan, A.H. (1997). Sur les chaos de Lévy stables d'indice $0<\alpha<1$. Ann. Sci. Math. Québec 21 53-66. MR1457064

[26] Gut, A. (2009). Stopped Random Walks: Limit Theorems and Applications, 2nd ed. Springer Series in Operations Research and Financial Engineering. New York: Springer. MR2489436

[27] Hammersley, J.M. (1974). Postulates for subadditive processes. Ann. Probab. 2 652-680. MR0370721

[28] $\mathrm{Hu}, \mathrm{Y}$. and Shi, Z. (2009). Minimal position and critical martingale convergence in branching random walks, and directed polymers on disordered trees. Ann. Probab. 37 742-789. MR2510023

[29] Kahane, J.-P. and Peyrière, J. (1976). Sur certaines martingales de Benoit Mandelbrot. Adv. in Math. 22 131-145. MR0431355

[30] Kingman, J.F.C. (1975). The first birth problem for an age-dependent branching process. Ann. Probab. 3 790-801. MR0400438

[31] Kyprianou, A.E. (2000). Martingale convergence and the stopped branching random walk. Probab. Theory Related Fields 116 405-419. MR1749282

[32] Liu, Q. (1998). Fixed points of a generalized smoothing transformation and applications to the branching random walk. Adv. in Appl. Probab. 30 85-112. MR1618888

[33] Lyons, R. (1997). A simple path to Biggins' martingale convergence for branching random walk. In Classical and Modern Branching Processes (Minneapolis, MN, 1994). IMA Vol. Math. Appl. 84 217-221. New York: Springer. MR1601749

[34] Lyons, R., Pemantle, R. and Peres, Y. (1995). Conceptual proofs of $L \log L$ criteria for mean behavior of branching processes. Ann. Probab. 23 1125-1138. MR1349164

[35] Madaule, T. (2011). Convergence in law for the branching random walk seen from its tip. Preprint. Available at http://arxiv.org/abs/1107.2543.

[36] Madaule, T. (2015). Maximum of a log-correlated Gaussian field. Ann. Inst. Henri Poincaré Probab. Stat. 51 1369-1431. MR3414451 
[37] Madaule, T., Rhodes, R. and Vargas, V. (2016). Glassy phase and freezing of log-correlated Gaussian potentials. Ann. Appl. Probab. To appear. Available at arXiv:1310.5574.

[38] Mandelbrot, B. (1974). Multiplications aléatoires itérées et distributions invariantes par moyenne pondérée aléatoire. C. R. Math. Acad. Sci. Paris 278 289-292. MR0431351

[39] Molchan, G.M. (1996). Scaling exponents and multifractal dimensions for independent random cascades. Comm. Math. Phys. 179 681-702. MR1400758

[40] Mörters, P. and Ortgiese, M. (2008). Minimal supporting subtrees for the free energy of polymers on disordered trees. J. Math. Phys. 49 125203, 21. MR2484334

[41] Ossiander, M. and Waymire, E.C. (2000). Statistical estimation for multiplicative cascades. Ann. Statist. 28 1533-1560. MR1835030

[42] Rhodes, R., Sohier, J. and Vargas, V. (2014). Levy multiplicative chaos and star scale invariant random measures. Ann. Probab. 42 689-724. MR3178471

[43] Shi, Z. (2016). Branching Random Walks. École D'été de Saint-Flour XLII. To appear.

[44] Stone, C. (1965). A local limit theorem for nonlattice multi-dimensional distribution functions. Ann. Math. Stat. 36 546-551. MR0175166

[45] Vatutin, V.A. and Wachtel, V. (2009). Local probabilities for random walks conditioned to stay positive. Probab. Theory Related Fields 143 177-217. MR2449127

[46] Waymire, E.C. and Williams, S.C. (1996). A cascade decomposition theory with applications to Markov and exchangeable cascades. Trans. Amer. Math. Soc. 348 585-632. MR1322959

[47] Webb, C. (2011). Exact asymptotics of the freezing transition of a logarithmically correlated random energy model. J. Stat. Phys. 145 1595-1619. MR2863721

Received October 2014 and revised October 2015 